\title{
Ejection of marine microplastics by raindrops: a computational and experimental study
}

\author{
Moritz Lehmann ${ }^{1 *}$ (D), Lisa Marie Oehlschlägel ${ }^{2}$, Fabian P. Häusl ${ }^{1}$, Andreas Held ${ }^{2}$ and Stephan Gekle ${ }^{1}$
}

\begin{abstract}
Raindrops impacting water surfaces such as lakes or oceans produce myriads of tiny droplets which are ejected into the atmosphere at very high speeds. Here we combine computer simulations and experimental measurements to investigate whether these droplets can serve as transport vehicles for the transition of microplastic particles with diameters of a few tens of $\mu \mathrm{m}$ from ocean water to the atmosphere. Using the Volume-of-Fluid lattice Boltzmann method, extended by the immersed-boundary method, we performed more than 1600 raindrop impact simulations and provide a detailed statistical analysis on the ejected droplets. Using typical sizes and velocities of real-world raindrops - parameter ranges that are very challenging for 3D simulations - we simulate straight impacts with various raindrop diameters as well as oblique impacts. We find that a $4 \mathrm{~mm}$ diameter raindrop impact on average ejects more than 167 droplets. We show that these droplets indeed contain microplastic concentrations similar to the ocean water within a few millimeters below the surface. To further assess the plausibility of our simulation results, we conduct a series of laboratory experiments, where we find that microplastic particles are indeed contained in the spray. Based on our results and known data - assuming an average microplastic particle concentration of 2.9 particles per liter at the ocean surface - we estimate that, during rainfall, about 4800 microplastic particles transition into the atmosphere per square kilometer per hour for a typical rain rate of $10 \frac{\mathrm{mm}}{\mathrm{h}}$ and vertical updraft velocity of $0.5 \frac{\mathrm{m}}{\mathrm{s}}$.
\end{abstract}

Keywords: Microplastics, Ocean, Atmosphere, Transport, Raindrop, Sea spray, Lattice Boltzmann method, Volume-of-Fluid, GPU

\section{Introduction}

Large water basins such as oceans or lakes are commonly considered as sinks where microplastic produced on land surfaces will accumulate over time [1-4], especially in coastal waters [5]. Atmospheric winds, on the other hand, can act as efficient transporters of microplastic leading to long-range, in fact even global, redistribution of atmospherically suspended microplastic [6-9]. Taken together, these two observations suggest that a mechanism transporting microplastic from the hydro- to the atmosphere might contribute significantly to the global spreading of

*Correspondence: moritz.lehmann@uni-bayreuth.de

'Biofluid Simulation and Modeling - Theorethische Physik VI, University of

Bayreuth, Bayreuth, Germany

Full list of author information is available at the end of the article microplastic. Indeed, hydrodynamic processes such as bursting bubbles [10-13] or breaking waves eject myriads of small water droplets into the air thus constituting an important mechanism for the transport of sea salt [14], organic material $[15,16]$ or particles $[17,18]$ which can have sizes up to $100 \mu \mathrm{m}$ [19]. It is to be expected that these processes are most relevant for particles near the ocean surface [20-23] and especially those that accumulate directly at the surface due to hydrophobicity, low density and/or bubble scavenging [24] as is very often the case for microplastic [25-27]. Indeed, evidence has recently been provided that these transport mechanisms could be relevant for microplastic as well $[7,28]$.

Another droplet-producing mechanism that has received much less attention is the impact of raindrops 
onto ocean or lake surfaces. Upon contact of the raindrop with the water surface, a thin wall of fluid around the impact site shoots upward at high speed. Due to hydrodyncamic instabilities, any distortion in this ring of fluid amplifies, leading to an uneven breakup into small droplets that resembles a crown in appearance. Depending on the raindrop diameter which is between 1 and $7 \mathrm{~mm}$ [29-33], each impact can eject more than hundred droplets during the initial splash. Quantifying this process is therefore crucial to understand if and how raindrop impacts can act as a possible pathway for microplastic transition from the hydro- into the atmosphere. While there are many experimental observations on various types of drop impacts into water [34-44] some even investigating particle transport [45] - significantly fewer works study raindrop impacts at terminal velocity [46-48]. This may be due to the fact that the drop height required to reach terminal velocity is several meters [30, 49]. Similarly, numerical simulations treating impact scenarios in the parameter range relevant for raindrops are also limited [50].

Here, we use a novel state-of-the-art GPU implementation of the Volume-of-Fluid lattice Boltzmann method (LBM), combined with the immersed-boundary method [51-53]. The LBM is a powerful tool for simulating fluid flow in countless fields such as microfluidics for medical applications and engineering; we use it due to its exceptional computational efficiency on graphics processing units (GPUs). We simulate more than 1600 impacts of raindrops with different diameters and impact angle, an amount unfeasible with other computational methods. We include microplastic of varying densities into the simulations to examine the potential for ejection. For each setting, we determine the size, altitude and airborne time distribution of droplets and ejected microplastic particles. In addition, we conduct laboratory experiments demonstrating the presence of microplastic particles in splash droplets after the impact of an artificial "raindrop" in good qualitative agreement with our simulations.

Based on our observations for single raindrops of different diameters, the raindrop size distribution [31, 33], typical microplastics concentrations in sea surface water $[3,54]$, precipitation data $[55,56]$ and typical vertical wind speeds close to the ground [57], we estimate the amount of microplastics that transition from the global oceans into the atmosphere annually due to raindrop impacts.

\section{Methods}

\section{Fluid solver}

\section{The lattice Boltzmann method}

For solving the Navier-Stokes equations, we use the simulation software FluidX3D [51-53], a full (multi) GPU implementation [53, 58-65] of the lattice Boltzmann method [66-68] which we thoroughly validated in-house (see SI section S2 and [51]). We use the single-relaxationtime collision operator [66], as both two-relaxation-time $[66,69]$ and multiple-relaxation-time [70-72] turned out to be unstable at such high Reynolds numbers in combination with Volume-of-Fluid. Gravity is incorporated using the Guo forcing scheme [66, 73]. The simulations are done in single-precision (FP32) floating-point.

\section{The Volume-of-Fluid model}

FluidX3D contains a full GPU implementation of the Volume-of-Fluid (VoF) model [51, 52, 74-79]. VoF introduces three flag types for LBM lattice points: fluid, interface and gas. The fluid phase is computed with regular LBM, the interface is kept sharp (width of a single lattice point) at any time and the gas phase is not calculated at all. On the interface layer, surface tension is handled based on the Young-Laplace equation and surface curvature, which is calculated using the paraboloid fit method [51, 74, 80], built upon the full analytic solution to the piecewise linear interface construction (PLIC) problem [52, 80-83].

\section{Hardware and illustrations}

Simulations are performed in parallel on up to four AMD Radeon VII GPUs (16 GB video memory each) as well as on a Nvidia Titan Xp GPU (12 GB video memory). With our efficient GPU implementation, the compute time for one impact simulation at $L_{x}=464$ on a single Radeon VII is between 5 and 15 minutes depending on data acquisition.

For grahpical illustrations, we use an OpenCL implementation of the marching-cubes algorithm [51, 84] that has direct read-only access to the raw simulation data in video memory. This way, rendering is fully parallelized on the GPU. On multiple GPUs, each one renders its own simulation domain and the individual images are stitched together based on their accompanying zbuffer. Lines in the images indicate the multi-GPU domain boundaries.

\section{Microplastic particles}

\section{The immersed-Boundary method}

The immersed-boundary method (IBM) $[51,85,86]$ couples particles to the fluid and is implemented fully parallelized on the GPU using trilinear velocity interpolation [87] (no-slip condition) and floating-point atomic addition [88]. IBM ensures proper two-way coupling between particles and fluid, whilst allowing particles to move freely between LBM lattice points. Each IBM particle corresponds to one microplastic particle.

\section{Particle properties}

The individual IBM particles have no coupling forces among each other, but they are buoyant and also prohibited from leaving the water and getting into the air. This is realized with a hard potential perpendicular to the local 
surface normal for particles between interface and gas VoF lattice points.

The particles are mathematical points without an intrinsic size. However there is a length scale of hydrodynamic interaction - the distance between two neighboring LBM lattice points converted back into SI-units - providing an upper bound for the particle diameter. Since the physical grid distance is adjusted to the diameter of the impacting raindrop, this upper limit for the microplastic particles $d_{p}$ is directly proportional to the raindrop diameter $d$ and varies between $d_{p} \lesssim 22 \mu \mathrm{m}$ and $d_{p} \lesssim 151 \mu \mathrm{m}$ for the $1 \mathrm{~mm}$ and $7 \mathrm{~mm}$ raindrops, respectively. This represents a realistic scenario as particles between $50-80 \mu \mathrm{m}$ are especially abundant in nature [3]. More details are given in S1.1.

\section{Analysis \\ Droplet detection}

Whenever a droplet touches the ceiling of the simulation box (i.e. in the uppermost lattice layer at least one lattice point becomes either interface or fluid), a Hoshen-Kopelman tracking algorithm [89] is triggered and all droplets in the simulation box are indexed. The droplets touching the ceiling are identified, measured and removed. Then the simulation continues until the next trigger event is detected.

Velocity $\vec{u}_{0}$ and radius $R_{0}$ are provided by the tracking algorithm for each droplet individually at the moment when the droplet touches the ceiling. For all fluid and interface lattice points belonging to a droplet, we average the velocity and calculate $R_{0}=\sqrt[3]{\frac{3 V_{0}}{4 \pi}}$ from the volume $V_{0}$.

\section{Estimating time of droplet separation from crown and cut-off altitude $h_{\text {cut }}$}

Our droplet detection algorithm delivers the time at which a droplet touches the ceiling of the simulation box. The physically relevant quantity, however, is the actual time of separation from the crown splash which we compute from the velocity and time at the moment of detection as described in the SI in section S1.2.

Because our simulations only cover the initial $10 \mathrm{~ms}$ of the splashing, only the droplets within this time frame are detected. It is an important observation that the first droplets that separate from the crown are the fastest, and later separating droplets are slower (Figure S3 (b)). We thus from our data define a cut-off altitude $h_{\text {cut }}$ in Figure S3 (b). After the simulated time frame, we expect only insignificant numbers of droplets to be ejected above $h_{\text {cut }}$. This gives us a threshold of confidence: below $h_{\text {cut }}$, almost all droplets are counted whereas above $h_{\text {cut }}$ some droplets could turn up after the simulation has finished. In the cumulative distributions of maximum altitude in this work, we mark $h_{\text {cut }}$ with a colored asterisk $*$, and we mark the regime of incomplete data, for that the cumulative distribution is considered the lower bound and the upper bound is undefined, as shaded areas.

\section{Trajectory of spray droplets: maximum altitude, airborne time and pickup by wind}

An impacting raindrop can eject a multitude of small droplets into the air, each of which may contain microplastic particles dispersed in the ocean water. If the microplastic particles are to be transported further into the atmosphere, pickup of these droplets by wind as well as their subsequent evaporation are essential. While we cannot simulate the maximum altitude of ejected droplets directly in our LBM simulation, since this would require very large simulation boxes, we can nevertheless calculate their $3 \mathrm{D}$ trajectories based on the initial position and velocity, initial drop radius $R_{0}$ at the moment of droplet detection as well as air properties. Although the droplets are tiny in size (with the minimum size resolvable in our simulations being $(\approx 233 \mu \mathrm{m}))$, their velocity is several meters per second, so Reynolds numbers are in the range $20-400$ (see Figure S4) and simple Stokesian friction does not apply in the first part of the trajectory [90] (see Figure S5). We therefore use the more general drag force model [91, p.116; 92]

$$
\vec{F}_{\text {drag }}=-\frac{1}{2} \rho_{\mathrm{a}} A C_{D}|\vec{u}| \vec{u}=-\beta C_{D}|\vec{u}| \vec{u}
$$

with $\rho_{\mathrm{a}}$ being the air density and $A=\pi R^{2}$ being the crosssection of a (spherical) droplet with radius $R . \vec{u}=\vec{u}(t)$ is the $3 \mathrm{D}$ velocity of the droplet. The parameter $\beta$ and the droplet mass $m$ can be written as functions of $R$ :

$$
\begin{aligned}
& \beta(R)=\frac{1}{2} \rho_{\mathrm{a}} A=\rho_{\mathrm{a}} \frac{\pi}{2} R^{2} \\
& m(R)=\rho \frac{4 \pi}{3} R^{3}
\end{aligned}
$$

$C_{D}=C_{D}(\operatorname{Re}(|\vec{u}|, R))$ is the drag coefficient for a viscous droplet with dynamic viscosity contrast as provided by Feng [92] (see section S1.3 in the SI). Re is the Reynolds number

$$
\operatorname{Re}(|\vec{u}|, R)=\frac{2 R \rho_{\mathrm{a}}|\vec{u}|}{\mu_{\mathrm{a}}}
$$

and $\mu_{\mathrm{a}}$ is the dynamic viscosity of air. Combining drag and gravity forces, the total force $\vec{F}$ on an airborne droplet is

$$
\begin{aligned}
m \dot{\vec{u}} & =\vec{F}(t)=\vec{F}_{g}+\vec{F}_{\text {drag }} \\
& =-\left(m-m_{\mathrm{a}}\right) g \vec{e}_{z}-\beta C_{D}(\operatorname{Re}(|\vec{u}|))|\vec{u}| \vec{u}
\end{aligned}
$$

with $m_{\mathrm{a}}$ being the mass of air the droplet displaces (buoyancy) and $g$ being the gravitational acceleration. The equations of motion for an airborne droplet are 


$$
\begin{aligned}
& \frac{d x(t)}{d t}=u_{x}(t), \quad \frac{d u_{x}(t)}{d t}=-k C_{D}|\vec{u}| u_{x}, \\
& \frac{d y(t)}{d t}=u_{y}(t), \quad \frac{d u_{y}(t)}{d t}=-k C_{D}|\vec{u}| u_{y}, \\
& \frac{d z(t)}{d t}=u_{z}(t), \quad \frac{d u_{z}(t)}{d t}=-k C_{D}|\vec{u}| u_{z}-g_{r}
\end{aligned}
$$

with

$$
\begin{aligned}
g_{r} & =\left(1-\frac{m_{\mathrm{a}}}{m}\right) g=\left(1-\frac{\rho_{\mathrm{a}}}{\rho}\right) g \\
k(R) & =\frac{\beta(R)}{m(R)}=\frac{3}{8} \frac{\rho_{\mathrm{a}}}{\rho} \frac{1}{R} .
\end{aligned}
$$

On top of this basic trajectory model, we consider two additional effects: (i) evaporation and (ii) updraft. While the droplet is airborne, its radius decreases due to evaporation. To include this effect in our model, we assume $[90$, eq. (36)]

$$
R(t) \approx \sqrt{R_{0}^{2}-\frac{K t}{16}}
$$

with $R_{0}$ being the initial radius and

$$
K=q_{0} \Delta T\left(1+2 q_{1} R_{0}\right)=2.628 \cdot 10^{-10} \frac{\mathrm{m}^{2}}{\mathrm{~s}}
$$

being the evaporation constant [90, eq. (46)]. The parameters $q_{0}=90.63 \cdot 10^{-12} \frac{\mathrm{m}^{2}}{\mathrm{sK}}, q_{1}=0.004225 \cdot 10^{6} \frac{1}{\mathrm{~m}}$ and $\Delta T=2.9 \mathrm{~K}$ are interpolated from [90, table 5] at $20^{\circ} \mathrm{C}$ and $75 \%$ relative humidity, the approximate conditions at the ocean surface [93]. This model is a simplification of the model presented in [90] in that it does not account for the evaporation rate depending on droplet velocity. It directly yields the lifetime [90, eq. (52)]

$$
t_{\text {life }}=\frac{2}{q_{0} q_{1}^{2} \Delta T}\left(2 q_{1} R_{0}-\log \left(1+2 q_{1} R_{0}\right)\right) .
$$

Note that we also ignore solute effects on the evaporation rate in this simplified equation.

In order to include the effect of updraft into our model, an additional vertical wind velocity offset $u_{\text {updraft }}$ is introduced in Eq. 1

$$
\vec{F}_{\text {drag }}=-\beta C_{D}\left|\vec{u}-u_{\text {updraft }} \vec{e}_{z}\right|\left(\vec{u}-u_{\text {updraft }} \vec{e}_{z}\right)
$$

with $C_{D}=C_{D}\left(\operatorname{Re}\left(\left|\vec{u}-u_{\text {updraft }} \vec{e}_{z}\right|\right)\right)$. Assuming constant updraft velocity instead of considering turbulent air movement of course is a simplification and in nature the process is more complicated.

We integrate (6) together with the effects of evaporation and updraft numerically using Runge-Kutta-4 with a stepsize of $\Delta t=0.1 \mathrm{~ms}$ (without updraft) $\Delta t=10 \mathrm{~ms}$ (with updraft) for each one of the approximately 17000 spray droplets per 100-impact-data-set. As initial conditions, we use the position and velocity from the droplet detection algorithm in section "Droplet detection". From the integration we obtain the maximum altitude and airborne time. From the airborne time we derive a clear criterion of microplastic uptake into the atmosphere. Two uptake scenarios are possible: either the airborne time diverges due to high updraft in combination with small droplet size or the airborne time remains finite (i.e. the droplet would eventually fall back onto the surface) but larger than the droplet life time. In this latter case, we also consider the microplastic particles to be taken up by the atmosphere.

For our setup (sea water and air, temperature $T=20^{\circ} \mathrm{C}$ ) we have $g=9.81 \frac{\mathrm{m}}{\mathrm{s}^{2}}, \rho=1024.8103 \frac{\mathrm{kg}}{\mathrm{m}^{3}}, \rho_{\mathrm{a}}=1.204 \frac{\mathrm{kg}}{\mathrm{m}^{3}}$ [94] and $\mu_{\mathrm{a}}=1.813 \cdot 10^{-5} \frac{\mathrm{kg}}{\mathrm{ms}}$ [95].

\section{Simulation setup and parameters}

Raindrops in nature are limited in diameter $d$ to approximately $1-7 \mathrm{~mm}$. If they are too small, they are mainly advected by winds, and if they are too large, they split into smaller droplets [33]. Depending on diameter and local air pressure, the terminal velocity (Table 1 ) is fixed $[29,30,32]$.

For simulating sea water, we use the kinematic shear vis$\operatorname{cosity} v=1.0508 \cdot 10^{-6} \frac{\mathrm{m}^{2}}{\mathrm{~s}}$, density $\rho=1024.8103 \frac{\mathrm{kg}}{\mathrm{m}^{3}}$ and surface tension $\sigma=7.381 \cdot 10^{-2} \frac{\mathrm{kg}}{\mathrm{s}^{2}}$ at standard temperature $T=20^{\circ} \mathrm{C}$ and standard absolute salinity $S=35 \frac{\mathrm{g}}{\mathrm{kg}}$ [96, 97]. The standard gravitational acceleration is $g=$ $9.81 \frac{\mathrm{m}}{\mathrm{s}^{2}}$. The raindrop fluid possesses the same parameters as sea water.

The depth of the liquid pool must be sufficient to approximate a 'deep' pool in that, during impact, the cavity expansion is not limited by the bottom wall of the simulation box. For a box deeper than a few times the drop diameter, there is no significant change in impact dynamics. In the simulation, the overall box size is limited by (video) memory, so a larger box size compared to the drop size will lower the resolution. We use a box of the size $10 d$ by $10 d$ by $8.5 d$ with a pool depth of $4 d$ as a compromise. The lateral boundaries are periodic and the very bottom lattice layer is a no-slip bounce-back boundary.

Table 1 Terminal velocity curve for raindrops at mean sea level pressure [29]

\begin{tabular}{lllllll}
\hline $\boldsymbol{d} / \mathbf{m m}$ & $\boldsymbol{u} / \frac{\mathrm{m}}{\mathbf{s}}$ & \multicolumn{1}{l}{$\boldsymbol{R e}$} & $\boldsymbol{W e}$ & $\boldsymbol{F r}$ & $\boldsymbol{C a}$ & Bo \\
\hline 1 & 4.50 & 4282 & 281 & 45.4 & 0.0657 & 0.136 \\
2 & 6.80 & 12943 & 1284 & 48.6 & 0.0992 & 0.545 \\
3 & 8.10 & 23125 & 2733 & 47.2 & 0.1182 & 1.226 \\
4 & 8.80 & 33498 & 4301 & 44.4 & 0.1284 & 2.179 \\
5 & 9.20 & 43776 & 5876 & 41.5 & 0.1342 & 3.405 \\
6 & 9.40 & 53673 & 7361 & 38.8 & 0.1371 & 4.903 \\
7 & 9.55 & 63618 & 8864 & 36.4 & 0.1393 & 6.674 \\
\hline
\end{tabular}

The terminal velocity $u$ is a function of the drop diameter $d$. The dimensionless numbers Reynolds $\operatorname{Re}=\frac{d u}{v}$, Weber $W e=\frac{d u^{2} \rho}{\sigma}$, Froude $F r=\frac{u}{\sqrt{d g}}$, Capillary $C a=\frac{u \rho v}{\sigma}$ and Bond $B O=\frac{d^{2} \rho g}{\sigma}$ are also given 
The microplastic particles are initialized at (reproducible) pseudo-random positions in the liquid pool with a concentration of 5000 particles per cubic centimeter several orders of magnitude higher than typically found in nature $(\approx 1-7 \text { particles per liter })^{1}$. If we chose typical particle concentration as found in contaminated sea water, there would be a single or no particle at all in the volume of the simulated domain. A particle concentration much higher would severely decrease computational efficiency as the buoyancy force of multiple nearby IBM particles would have to be atomic-added to each lattice point. Since our particles do not directly interact with each other and buoyancy effects are negligible at short time scales, the number of ejected particles per raindrop impact can be scaled down linearly with the initial particle concentration. The raindrop initially is devoid of particles.

During unit conversion from SI-units to lattice units, the density $\rho=1$ in lattice units is fixed and the length scale $L_{x}$ is limited by memory capacity. The impact velocity $u$ in lattice units however is a free parameter that does not change physics, but has large impact on both compute time (proportional to $\frac{1}{u}$ ) and accuracy. Tests with Poiseuille flow in a cylindrical channel showed that for FP32 floating-point accuracy, $u$ should be in the range $0.0003 \leq u \leq 0.5$ and especially $u \leq 0.5$ anywhere in the simulation box [51]. In Figure S12 in S3.1 we show that simulations with $u \in[0.005,0.15]$ run stable. Outside of this interval, instabilities propagate from the first unstable lattice point through the simulation box. We thus use $u=0.05$ for all of our simulations.

We also show simulations for varying lattice resolution $L_{x} \in\{64,128,256,464,636,748\}$ in S3.2 and observe that $L_{x}<400$ is insufficient to resolve details.

Simulations are run for a time span of $10 \mathrm{~ms}$. In nature, around $10 \mathrm{~ms}$ after impact low air pressure behind the falling droplet contracts the crown to a canopy or surface seal [46]. Since in our simulation we do not model the gas phase, we do not observe surface seal formation and thus for longer simulation periods our results would significantly divert from experimental findings. However only in the initial splash phase of the impact do ejected droplets have sufficiently small size and high velocity to be relevant for atmospheric pickup. Droplets ejected later are slower and more likely to fall back to the surface instead of contributing to transport across the interface. The later occurring jet in the experiment also does not significantly contribute to ejected high-velocity droplets $[41,46]$.

\footnotetext{
${ }^{1}$ Measurements of microplastic concentration on sea surface water greatly vary with time, location and measurement method, ranging from 0.0018 [20] over 0.04 [21] to 0.406 [22] microplastic particles per $\mathrm{m}^{2}$ in trawl measurements. [54] provide an average volumetric value of 2.9 particles per liter at $5 \mathrm{~m}$ depth and [3] provide measurements of between $0.99-7.00$ particles per liter across the Atlantic Ocean at $10 \mathrm{~m}$ depth.
}

\section{Simulation results}

Validating our simulations by comparison with experiment To begin our investigations, we validate the employed simulation model by comparing it to high-speed images of a raindrop impact in sea water. Further validation is provided in S2. Murphy et al. [46] studied oily marine aerosol production when raindrops impact oil slicks on the ocean. As a control, they conducted and documented a $4.1 \mathrm{~mm}$ diameter raindrop impact on pure seawater at an impact velocity of $7.2 \frac{\mathrm{m}}{\mathrm{s}}-\mathrm{a}$ bit less than terminal velocity. The fluid properties used in [46] (see Table S2 of the SI) coincide almost exactly with the ones used in our microplastic simulations as detailed below. This allows for a direct experimental validation of our simulation setup in the relevant parameter range. In Fig. 1, we compare the simulation results to the experiment finding excellent agreement for the cavity size and crown breakup. A deviation between experiment and simulation is only seen at $t=8 \mathrm{~ms}$ where the crown in the experiment contracts and begins to form a canopy. This is due to the lower air pressure behind the falling raindrop (Bernoulli effect) that pulls the crown inwards in what is commonly called a surface seal $[98,99]$. Since air flow is not explicitly included in our simulations, we do not see the crown contracting. In this work, however, we focus on the generation and ejection of small droplets which primarily happens in the beginning of splash formation and thus will not be significantly affected by the entraining air flow.

\section{Simulation of raindrop impacts on pure sea water}

We next illustrate our $4 \mathrm{~mm}$ diameter raindrop impact reference system in Fig. 2 for pure sea water without microplastic particles. The corresponding parameters are given in Table 2 . Shortly after impact, the perimeter of the impact site shoots upward. It forms a thin wall of fluid - called crown - that quickly breaks up into lots of tiny droplets due to surface tension. Crown droplets initially are small and fast with a velocity inclined by approximately $53^{\circ}$ from the vertical axis radially outward. As time progresses, they gradually become larger and slower (with less velocity inclination). Shortly after the raindrop and pool surfaces touch (time of impact), the upper half of the raindrop initially retains its convex shape which now forms the bottom surface of the impact cavity while displaced fluid exits upward at the perimeter. Once the raindrop fluid plunges further in - about $1 \mathrm{~ms}$ after impact - the cavity center flattens and becomes concave thereafter, steadily expanding while more and more fluid is pushed into the crown. The fluid of the raindrop spreads into a thin sheet around the cavity. During the simulated $10 \mathrm{~ms}$, the cavity and crown continuously expand while the gravitation-driven cavity collapse followed by the wellknown vertical fluid jet [41, 46, 100] happen only at a 

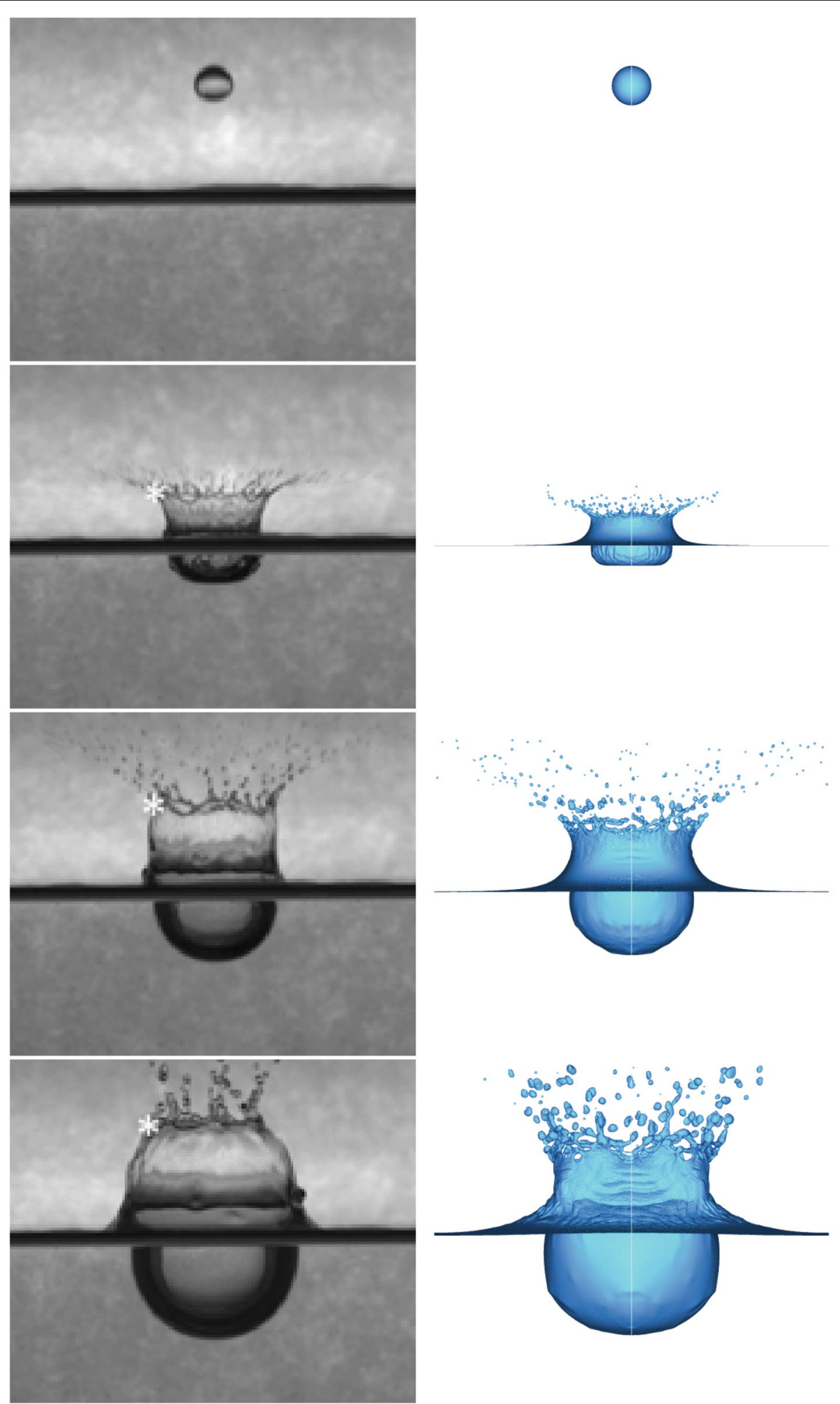

Fig. 1 Our simulation compared to the experiment from Murphy et al. [46], Fig. 3 (a)-(d), at times $t \in\{-2,1,3,8\}$ ms. In both experiment and simulation, $t=0 \mathrm{~ms}$ is defined as the time the droplet touches the water surface. The raindrop diameter is $4.1 \mathrm{~mm}$. The asterisk marks the tracked crown rim position in the experiment 

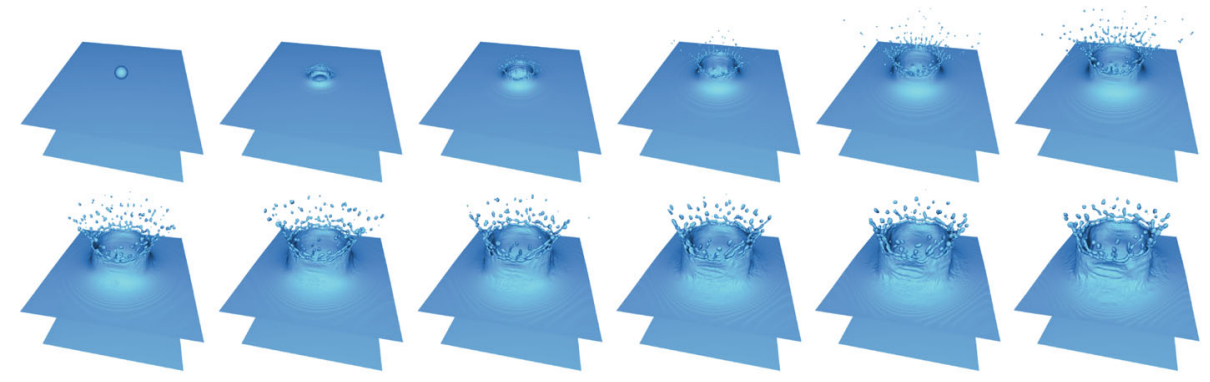

(a) Perspective view
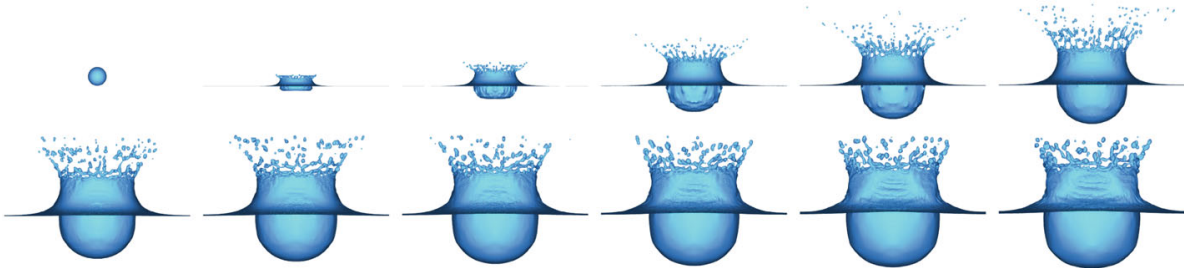

(b) Orthogonal side view.

Fig. 2 Visual representation of the $4 \mathrm{~mm}$ diameter raindrop impact simulation without microplastic particles. Time stamps (left to right) are $t \in\{0.0,0.5,1.0,2.0,3.0, \ldots, 10.0\} \mathrm{ms}$ and lattice resolution is $L_{x}=464$

later stage. The fastest droplets that reach the highest altitude in the air are ejected within the first few milliseconds after impact. Previous research has suggested that the secondary jet after cavity collapse does not contribute greatly to ejection of droplets $[41,46]$ so for this study we have not included it.

\section{Impacts of raindrops on sea water with microplastic particles}

We now include microplastic particles in the simulation. To obtain sufficient statistics of ejected droplets and particles, we run the simulation 100 times for each set of parameters, with the microplastic particles each time being initialized at different random positions, resulting in slightly different random crown breakup. We choose the highest possible lattice resolution for our IBM-LBM simulations $\left(L_{x}=464\right)$ and run each simulation for $10 \mathrm{~ms}$. Droplets that touch the top of the simulation box are measured and then deleted from the simulation box as described in section "Droplet detection" above. The upper bound for the microplastic diameter is $86 \mu \mathrm{m}$ as determined in $\mathrm{S} 1.1$ and their density is $1.05 \frac{\mathrm{g}}{\mathrm{cm}^{3}}$ (polystyrene). In Figure S23 we provide data on the velocity inclination from the vertical axis of the ejected droplets for the $4 \mathrm{~mm}$ diameter raindrop reference data set. In S4.7 we provide simulations over the entire range of common plastics densities from $0.92 \frac{\mathrm{g}}{\mathrm{cm}^{3}}$ (polypropylene) to $2.17 \frac{\mathrm{g}}{\mathrm{cm}^{3}}$ (polytetrafluoroethylene) and demonstrate that buoyancy effects are negligible during the simulated time frame. Figure S27 demonstrates how small the run-to-run

Table 2 Overview on the simulation parameters before and after unit conversion

\begin{tabular}{lll}
\hline & SI-units & LBM units \\
\hline drop diameter $d$ & $1-7 \mathrm{~mm}$ & 46.4 \\
impact velocity $u$ & $4.50-9.55 \frac{\mathrm{m}}{\mathrm{s}}$ & 0.05 (fixed) \\
impact angle $\alpha$ & $0^{\circ}-40^{\circ}$ & $0^{\circ}-40^{\circ}$ \\
simulation box dimensions $L_{x}, L_{y}, L_{z}$ & $10 d, 10 d, 8.5 d$ & $464,464,394($ fixed) \\
pool height $h$ & $4 d$ & 185.6 \\
kinematic shear viscosity $v$ & $1.0508 \cdot 10^{-6} \frac{\mathrm{m}^{2}}{\mathrm{~s}}$ & $5.417 \cdot 10^{-4}(1 \mathrm{~mm})-3.647 \cdot 10^{-5}(7 \mathrm{~mm})$ \\
water density $\rho$ & $1024.8103 \frac{\mathrm{kg}}{\mathrm{m}^{3}}$ & $1(\mathrm{fixed})$ \\
surface tension $\sigma$ & $7.381 \cdot 10^{-2} \frac{\mathrm{kg}}{\mathrm{s}^{2}}$ & $4.126 \cdot 10^{-4}(1 \mathrm{~mm})-1.309 \cdot 10^{-5}(7 \mathrm{~mm})$ \\
gravitational acceleration $g$ & $9.81 \frac{\mathrm{m}}{\mathrm{s}^{2}}$ & $2.610 \cdot 10^{-8}(1 \mathrm{~mm})-4.057 \cdot 10^{-8}(7 \mathrm{~mm})$ \\
(hydrodynamic) particle diameter $d_{p}$ & $\lesssim 22-151 \mu \mathrm{m}$ & $\approx 0.6-1.0(\mathrm{fixed})$ \\
number of particles $N_{p}$ & $2000-686000$ & $2000-686000$
\end{tabular}


variation is in the simulation results, giving us confidence about the accuracy of our data. In section S3.2 we provide data on the influence of lattice resolution on the droplet distribution.

\section{Microplastic transport during $4 \mathrm{~mm}$ diameter raindrop impacts}

Figure 3 shows how the ejected droplets of 100 raindrop impact simulations are distributed in diameter ( $x$-axis), ejection altitude ( $y$-axis) and time of detection (color).

We observe that droplets at later points in time have more volume, but less velocity, so maximum altitude is lower. After $t=5 \mathrm{~ms}$ almost no droplets are ejected beyond half a meter in amplitude. This gives us confidence that our simulations capture all droplets with a high ejection velocity which are relevant to microplastic transport. The vertical velocity of the small droplets ejected at an early stage reaches up to about $10 \frac{\mathrm{m}}{\mathrm{s}}$, while the velocities of larger droplets ejected at later times are below $3 \frac{\mathrm{m}}{\mathrm{s}}$. In Figs. 4 and 5, we present histograms to reveal the size distribution of droplets and the cumulative ejected fluid volume as well as the distribution of how many particles are present in droplets and the particle concentration depending on droplet diameter. The bin width in all histograms is $0.02 \mathrm{~mm}$.

We observe that, by number, the majority of ejected droplets has a diameter below $0.6 \mathrm{~mm}$ with the peak centered at $0.4 \mathrm{~mm}$. In reality, there are likely to be more smaller droplets because we cannot resolve droplets below $0.23 \mathrm{~mm}$ diameter in our simulation. Between 0.6 and $1.0 \mathrm{~mm}$, we observe a relatively flat plateau. These larger droplets are less numerous, but contribute more than double to cumulative ejected fluid volume than the small droplets as can be seen in Fig. 4 (b). Comparing Figs. 4 (b) and 5 (a), we find good proportionality between cumulative ejected fluid volume and the number of particles in the ejected droplets. Larger droplets with more fluid carry more particles. The particle concentration in ejected

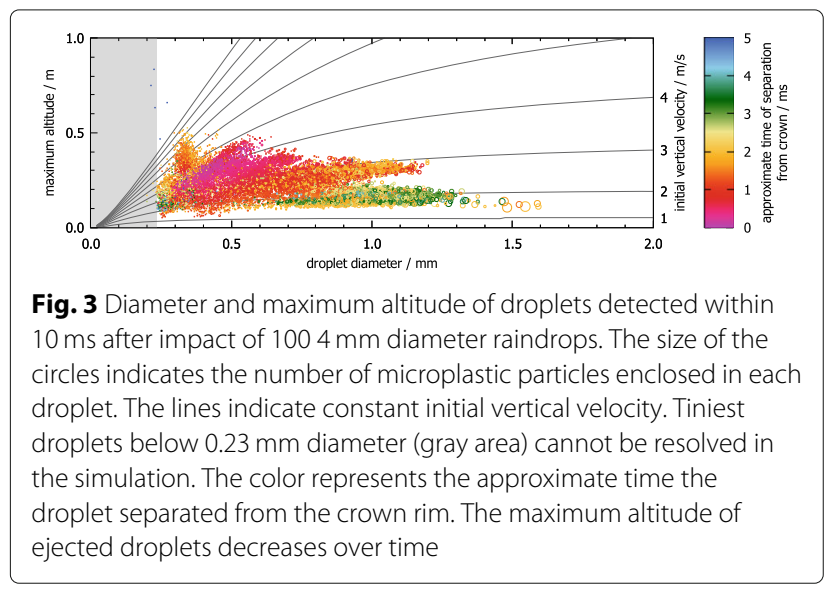

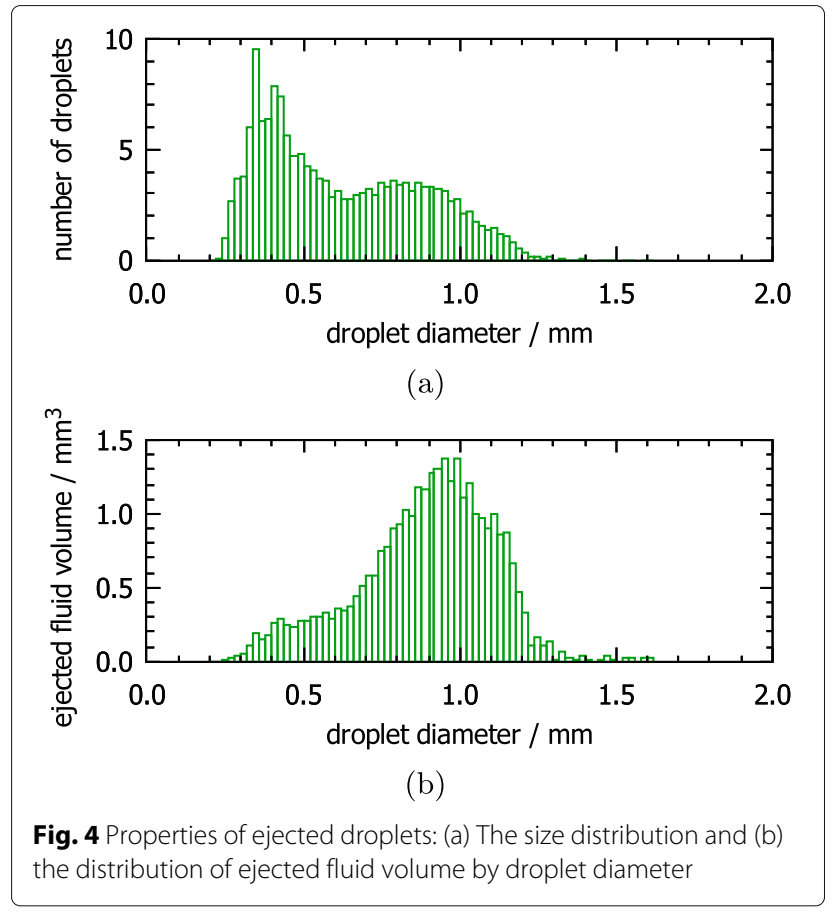

droplets is on average $87 \%$ compared to the initial concentration in the pool, meaning that the raindrop fluid makes up approximately $13 \%$ of the ejected fluid. Only for the smallest droplets, the particle concentration appears to be a bit lower, closer to $70 \%$ which most likely is an effect of numerical resolution and a larger uncertainty, as in this region there is very little total fluid volume to divide the particle number by.

To conclude this section, we find that a single $4 \mathrm{~mm}$ diameter raindrop ejects about 167 droplets $(>0.23 \mathrm{~mm}$ diameter $^{2}$ ) during the first $10 \mathrm{~ms}$ after impact. These droplets contain a total of 136 microplastic particles for an initial concentration of 5000 microplastic particles per $\mathrm{cm}^{3}$ in the sea water.

\section{How raindrop diameter affects microplastics transport}

The diameter of raindrops, which determines their terminal velocity according to Table 1 , strongly affects the number of ejected droplets and microplastic particles. Figure 6 shows the distribution of size, altitude and microplastic load of ejected droplets similar to our $4 \mathrm{~mm}$ reference system in Fig. 3, when the raindrop diameter varies between 2 and $7 \mathrm{~mm}$. Snapshots of the corresponding simulations are shown in Figure S28. From these visualizations we conclude that raindrops with diameters below $2 \mathrm{~mm}$, although frequently occuring in nature, do not eject a significant number of droplets into the air. Raindrops with diameters above $7 \mathrm{~mm}$ are very rare in natural rain events and are therefore not considered further. Note that the

\footnotetext{
${ }^{2}$ smaller droplets cannot be resolved in the simulation
} 


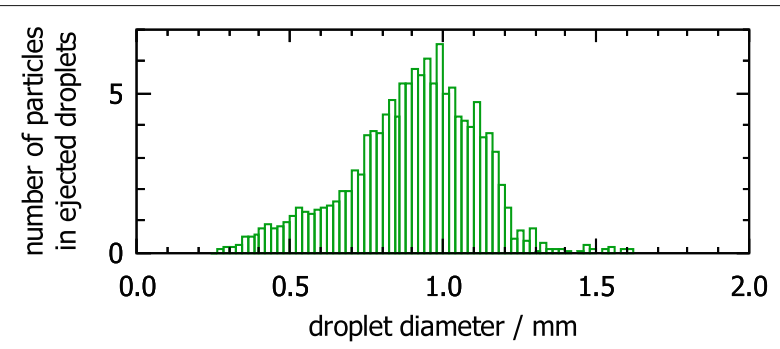

(a)

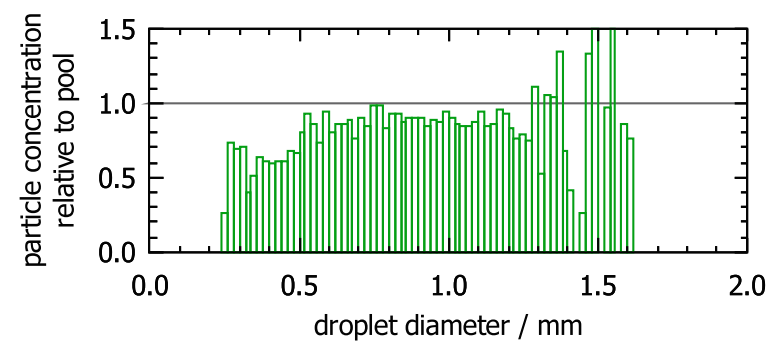

(b)

Fig. 5 Properties of microplastic particles in ejected droplets: (a) The distribution of particles in droplets and (b) particle concentration depending on droplet diameter

minimum resolvable droplet size is larger for simulations of larger raindrops and thus the gray area in Fig. 6 where no droplets can be resolved, increases.

For a more quantitative description, Fig. 7 (a) provides histograms of the droplet size distribution for different raindrop diameters. We observe that with increasing raindrop diameter, the average size of produced droplets increases simultaneously. Figure 7 (b) shows a similar trend for the number of microplastic particles ejected by droplets of various sizes: for larger raindrops the contribution of the larger droplets increases. We furthermore note that similar to the $4 \mathrm{~mm}$ reference case of the previous section, within the droplets we find a constant particle concentration of on average $85 \%$ the bulk value regardless of raindrop diameter (data not shown).

In the SI in Figure S3 we provide the maximum altitude of ejected droplets as function of the time the droplets separate from the crown rim. We see that - despite the impact velocity being larger for bigger raindrops - the overall time scale of the impact increases with droplet size. Figure S3 furthermore shows that the maximum altitude of the ejected droplets decreases monotonically over time. This illustrates an important limitation of our simulations: since we only consider the first $10 \mathrm{~ms}$ after impact, droplets after this time are missing in our statistics. For small raindrops with sizes up to $4 \mathrm{~mm}$, Figure S3 shows that the amount of missed droplets can be expected to be small and, more importantly, the maximum altitude of the missing droplets will not be larger than a cut-off of around

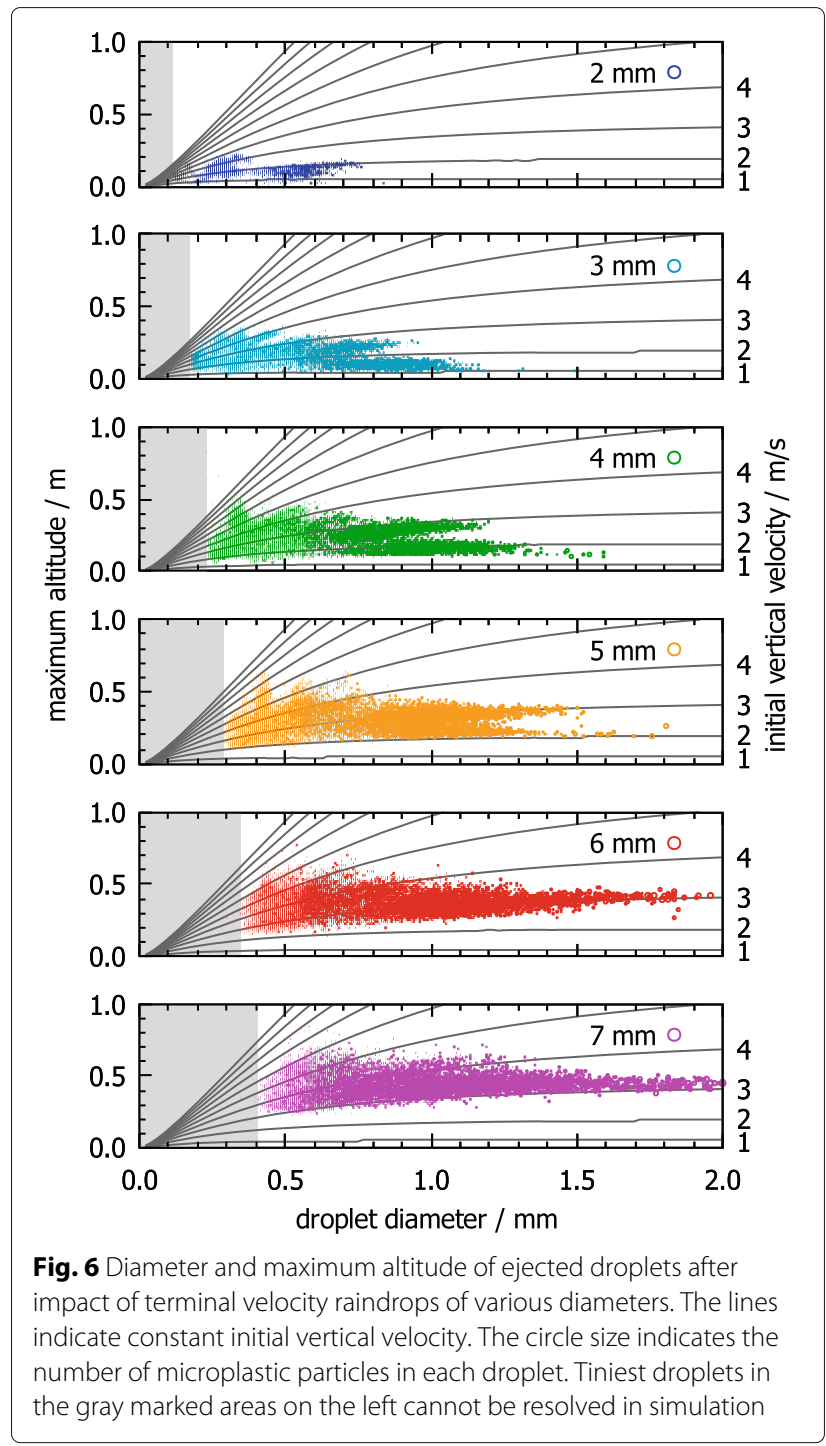

$h_{\text {cut }}=0.19 \mathrm{~m}$. This picture changes for raindrops with diameters above $4 \mathrm{~mm}$, where a substantial amount of droplets are missed. Nevertheless, Figure S3 allows us to determine $h_{\text {cut }}$ depending on raindrop size. For the largest raindrops of $7 \mathrm{~mm}, h_{\text {cut }}=0.48 \mathrm{~m}$, meaning that the vast majority of droplets ejected to altitudes above $0.48 \mathrm{~m}$ are counted, and some droplets with ejection altitudes below $0.48 \mathrm{~m}$ are missed.

In Fig. 8 we show the number of ejected droplets and number of particles in ejected droplets as a function of maximum altitude. The droplets from the initial phase of the impact are very small and therefore carry less particles than droplets with larger volume from the later phase of the impact, so the cumulative distribution of maximum altitude of the particles drops steeper than that of the droplets. 


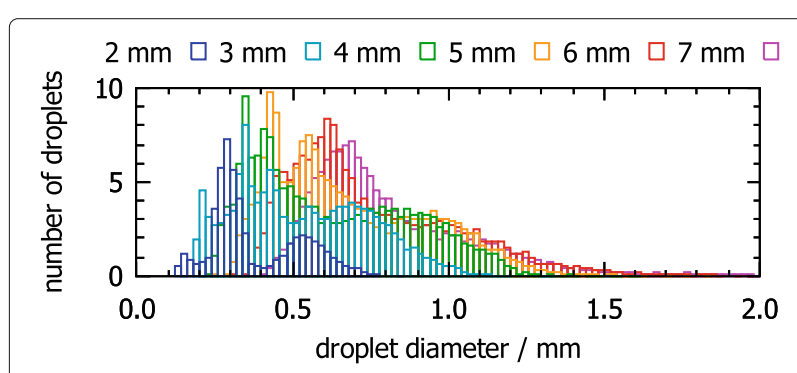

(a)

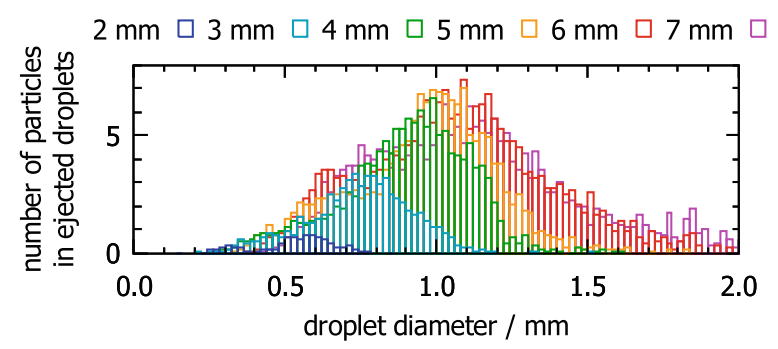

(b)

Fig. 7 (a) Size distribution of ejected droplets and (b) distribution of particles in ejected droplets depending on droplet size for various raindrop diameters. In the $\mathrm{SI}$, we show these histograms as separate plots for each raindrop diameter in Figures S21 and S22

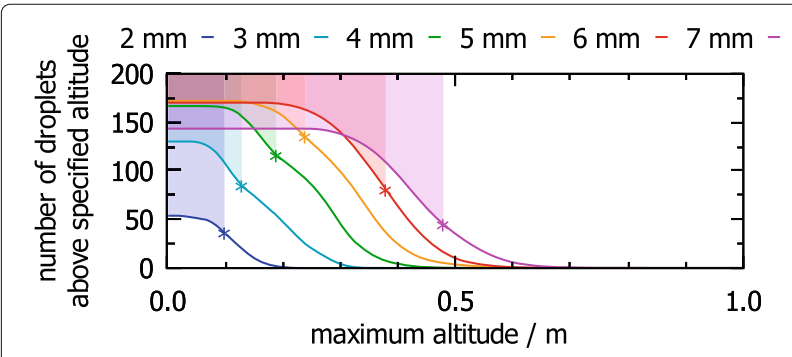

(a)

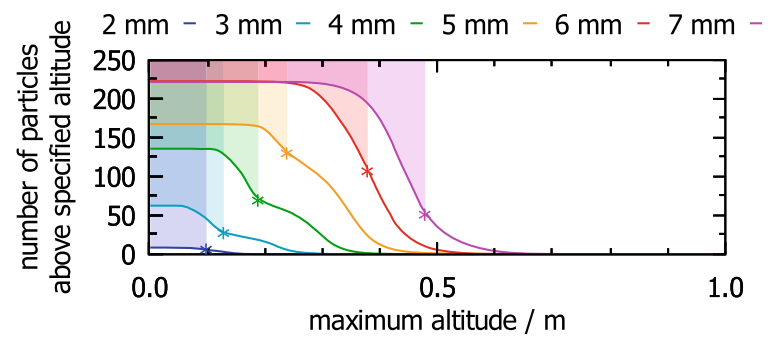

(b)

Fig. 8 Cumulative distribution of maximum altitude of resolvable droplets (a) and microplastic particles in droplets (b). The graphs indicate the number of droplets / particles ejected above a specified altitude depending on raindrop diameter. Above the cut-off altitude $h_{\text {cut }}$ marked by the asterisk, all droplets are detected by our simulations (solid line). Below $h_{\text {cut }}$, our simulations only provide a lower bound for the number ejected droplets (dashed line) due to limited simulation time as explained in section "Estimating time of droplet separation from crown and cut-off altitude $h_{\text {cut }}{ }^{\prime \prime}$

\section{How oblique impacts affect microplastics transport}

Due to influence of wind, it may be expected that most raindrops will not fall perfectly straight and will impact the water surface at an angle. Such oblique impacts have an inclined and asymmetric crown geometry as illustrated in Fig. 9 and in Figures S29 and S30 in the SI. This affects how the fluid of the raindrop, which is initially devoid of microplastics, is distributed in the bulk fluid during impact. For the straight impact, the raindrop water is spread around the bottom of the cavity as a thin film and not ejected into the air as demonstrated by the high microparticle concentration in the droplets in Fig. 5 (b). This behavior changes for oblique impacts. As shown in Fig. 10 (a), here the raindrop fluid is partly redirected into the crown, thus slightly reducing the overall particle concentration in the ejected droplets from $87 \%$ of the pool concentration $\left(\alpha=0^{\circ}\right)$ to $77 \%\left(\alpha=40^{\circ}\right)$.

Besides this, there is a second, equally interesting effect emerging: For the straight impact, the fast droplets directly after impact are ejected radially outward with their velocity inclined from the vertical axis as can be seen in the first frames of Fig. 2. Thus, on one side the trajectory of the small droplets becomes more vertical, resulting in more droplets being ejected to higher altitude as shown in Fig. 10 (b). In addition, the total amount of ejected droplets during our simulated $10 \mathrm{~ms}$ time frame also increases. For this reason, the initial fast droplets carry microplastic particles to higher altitudes for oblique impacts. Nevertheless, since the water from the raindrop itself is partly deflected into the crown later on, the total amount of ejected particles is actually smaller for oblique impacts as shown in Fig. 10 (c).

\section{Origin of ejected microplastic particles}

Using our simulations, we can also detect from which region of the bulk fluid (relative to the impact location) the microplastic particles originate that are ejected into the air. For this, we assign a unique ID number to each microplastic particle in the bulk fluid and then store the IDs of those particles that are ejected into the air during the impact simulation of $10 \mathrm{~ms}$. The initial position of those particles is marked in red in Fig. 11. We find that only particles from the very top layer of the pool are ejected in the crown. The initial positions furthermore form a ring around the impact site.

Figures S28 and S30 illustrate particle origin for various raindrop diameters and impact angles. In all cases only particles directly on the water surface or a few millimeters below the surface are relevant for consideration.

\section{Experimental demonstration of microplastic transport}

In addition to the detailed simulations, we conduct a series of laboratory experiments demonstrating microplastic 

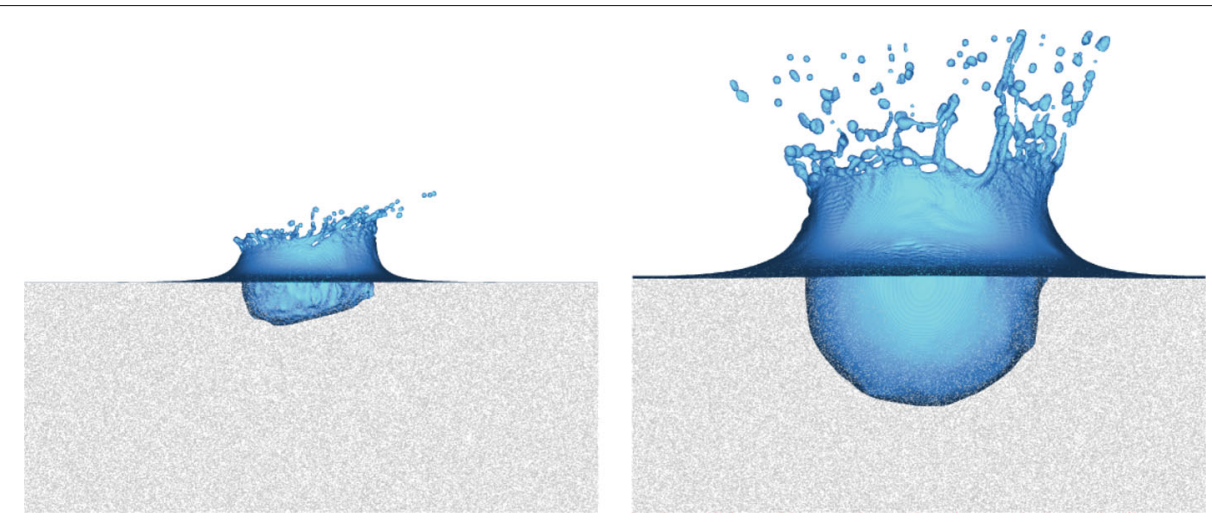

Fig. 9 Illustration of a $\alpha=20^{\circ}$ inclined raindrop impact at times $t \in\{1,5\} \mathrm{ms}$

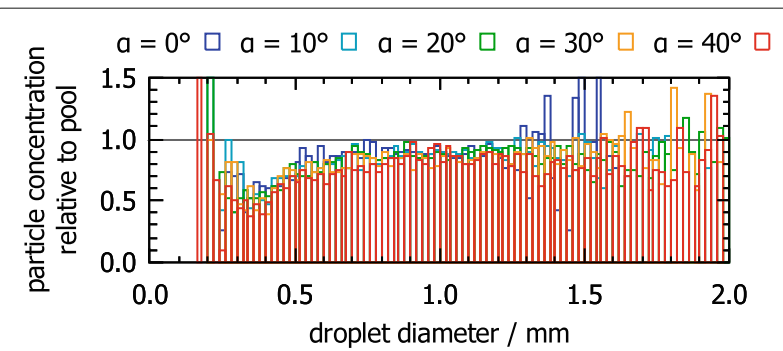

(a)

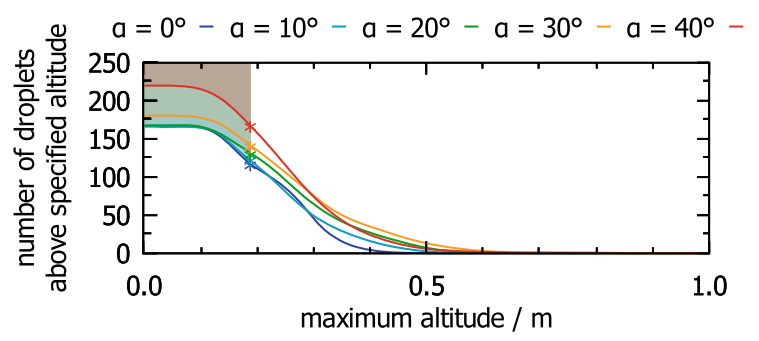

(b)

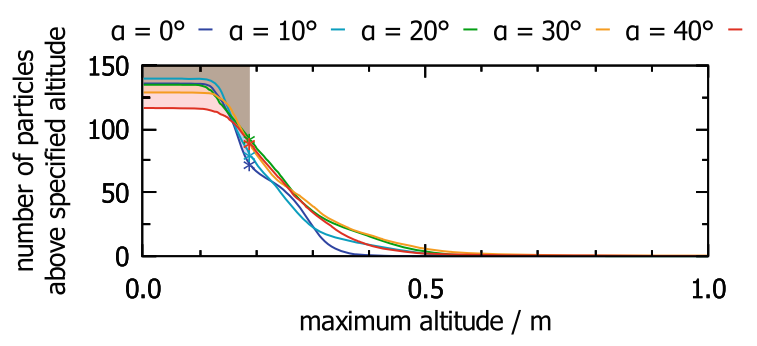

(c)

Fig. 10 Simulation results for oblique impacts: (a) Distribution of particle concentration in ejected droplets depending on droplet size and impact angle $\alpha$ as well as (b) number of resolvable droplets and (c) particles in droplets ejected above a specified altitude depending on impact angle $\alpha$. In (b) and (c), for altitudes lower than $h_{\text {cut }}$ (asterisks), the distributions are considered the lower bound (dashed lines) and the upper bound is undefined as indicated by the shaded areas (see section "Estimating time of droplet separation from crown and cut-off altitude $\left.h_{\text {cut }}{ }^{\prime \prime}\right)$ transport by impacting drops. An image of our experimental setup is shown in Fig. 12 (a). The setup consists of a timer-controlled magnetic valve (eltima electronic) which allows us to adjust the size of the falling drop by changing the opening time of the valve. A Mariotte's bottle containing desalinated water is connected to the valve with a nozzle below. The surface tension of desalinated water $\left(72.75 \frac{\mathrm{mN}}{\mathrm{m}}\right)$ only insignificantly differs from that of sea water $\left(73.39-76.67 \frac{\mathrm{mN}}{\mathrm{m}}\right.$ depending on salinity) at $20^{\circ} \mathrm{C}$. We note that natural surfactants in the ocean surface microlayer, enriched by for example bubble scavenging [24], may slightly decrease surface tension compared to pure salt water. In our experiments, microplastic particles could, in principle, reduce surface tension in a similar fashion. However, the volumetric ratio of microplastics to water is $\approx 10^{-6}$ and thus small enough that this effect can safely be neglected regarding both viscosity ${ }^{3}$ and surface tension. The drop falls into a water reservoir which is filled with desalinated water and spherical polystyrene particles with a diameter of $6 \mu \mathrm{m}$ (Polysciences). The particle concentration in the reservoir is $25000 \frac{1}{\mathrm{~cm}^{3}}$. When opening the magnetic valve for $20 \mathrm{~ms}$, we obtain falling drops with a diameter of approximately $5.0-5.5 \mathrm{~mm}$. Similar to the simulation, the water height in the reservoir is about 4 times the drop diameter. The distance between the water body and the nozzle exit is approximately $2.5 \mathrm{~m}$, based on which we estimate the impact velocity to be $6.8 \frac{\mathrm{m}}{\mathrm{s}}$ [49], i.e. about $74 \%$ of terminal velocity [29]. The splash droplets released after impact are caught on a $76 \mathrm{~mm} \times 26 \mathrm{~mm}$ glass slide mounted $20 \mathrm{~cm}$ above the reservoir. The glass slide is placed such that the inner $26 \mathrm{~mm}$ edge is $65 \mathrm{~mm}$ (position A) and $141 \mathrm{~mm}$ (position B) radially outward from the impact center (Fig. 12 (c)).

After the impact, the glass slide is transferred to a microscope immediately. Although the glass slides are carried openly to the microscope located in the same room and

${ }^{3}$ Einstein suspension viscosity $[101,102]$ is $0.0007 \%$ larger than without particles. 


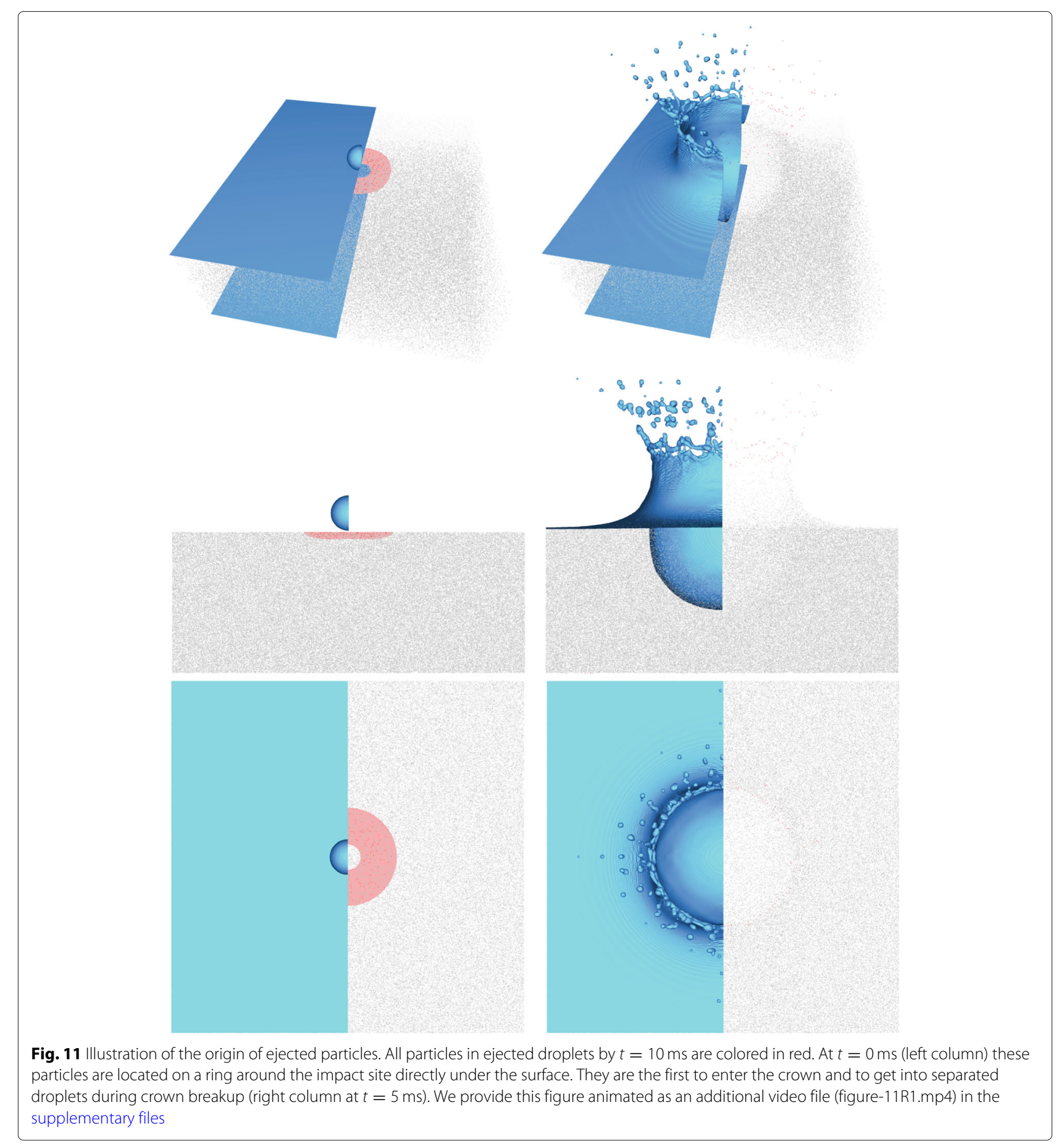

thus a small contamination by particles from room air is possible, it would be highly unlikely that contaminant particles coincidentally possess the same uniform diameter $(6 \mu \mathrm{m})$ as our microplastic particles. The size and spherical shape of the counted particles thus clearly shows that the observed particles on the glass side are indeed microplastic ejected from the liquid reservoir. Independent of the rain drop experiments we control the shape and size of our polystyrene particles by attenuating the suspension containing the particles and assessing them under the microscope to confirm the spherical shape and the diameter of $6 \mu \mathrm{m}$.

Using the microscope we clearly observe the presence of microplastic particles in the splash droplets (Fig. 12 (b)). For positions A and B we conduct 10 drop impacts each. We then count the number of droplets on the glass slide 


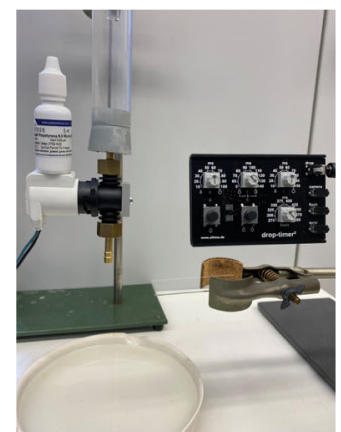

(a)

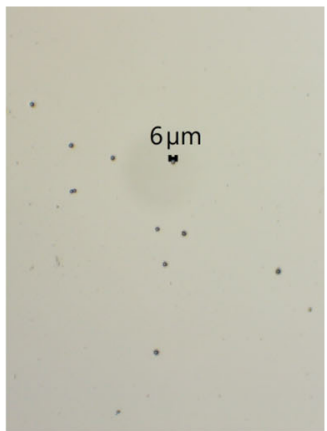

(b)

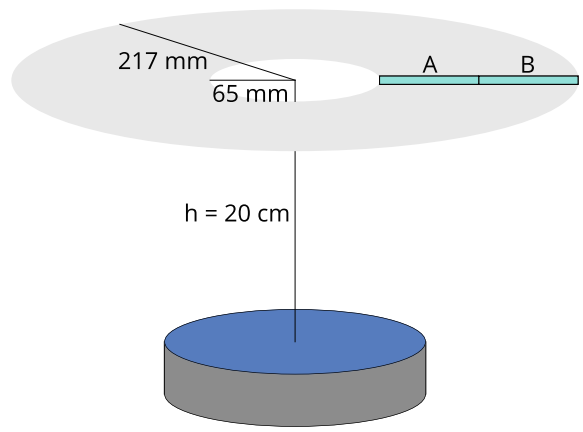

(c)

Fig. 12 (a) The experimental setup with timer-controlled magnetic valve for the creation of individual drops, connected to a Mariotte's bottle above and a nozzle below. Released drops impact on a reservoir filled with desalinated water and $6 \mu \mathrm{m}$ spherical polystyrene particles. Ejected droplets are captured on a glass slide. Note that for illustration purposes, valve and reservoir are shown together, while in our experiments the falling distance is $2.5 \mathrm{~m}$. (b) Splash droplets carry particles to a glass slide placed above the reservoir. The droplets evaporate, leaving the microplastic particles behind. (c) Sketch of the glass slide placement (positions A and B) above the reservoir. The radial symmetry of the impact splash allows to only cover a circle segment with the glass slides and extrapolate to the annular cross-section areas around the A and B placements (gray rings) through which ejected droplets may pass. We provide high-speed video of the experiment in subfigure (a) as an additional video file (figure-12aR1.mov) in the supplementary files

that have not yet evaporated, measure their approximate diameter, their position on the slide and the number of particles inside. The number of tested splash drops depends on the number of drops found on the glass slide and how many of the drop positions could be clearly identified under the microscope. Some of them evaporated before their location on the slide was noted down. Some of the ejected droplets do not contain any plastic particles. In some cases, we could test all droplets for microplastic particles if there were only between 1 and 3 droplets on the slide.

We find that for a single drop impact, the average number of droplets on the glass slide at position $\mathrm{A}$ is 4.2 and on position $B$ is 2.4. The average number of particles per detected droplet is 16 and 3 for positions $A$ and $B$, respectively. The difference in particles per droplet for positions $A$ and $B$ is due to the smaller average size of the droplets at $B$. This is in full agreement with the numerical simulations: as can be seen in Figure S23, the initially released small droplets have a higher velocity inclination from the vertical axis and thus are more likely to land on the glass slide at position $\mathrm{B}$, whereas larger droplets released at later times have a smaller velocity inclination and are more likely to land at position A.

In order to obtain the total number of ejected droplets by a single drop impact, we first extrapolate the area of the glass slides at $\mathrm{A}$ and $\mathrm{B}$ to the two cross-sectional rings around positions $\mathrm{A}$ and $\mathrm{B}$ (Fig. 12 (c)). In addition we re-scale the particle concentration to $5000 \frac{1}{\mathrm{~cm}^{3}}$ to be able to directly compare with the simulation. Adding the two cross-sectional rings $\mathrm{A}$ and $\mathrm{B}$, we find that 208 droplets containing 398 particles are ejected to an altitude of $20 \mathrm{~cm}$ or higher. The number of droplets is in very good agreement with the simulations (approximately 1.3 times larger) as can be seen in Fig. 8 (a) when looking at the lower bound of the shaded area for the $5 \mathrm{~mm}$ curve at $0.2 \mathrm{~m}$ altitude. The number of particles, on the other hand, is approximately 2.5 times larger in the experiment than in our simulations (Fig. 8 (b), lower bound of the shaded area for the $5 \mathrm{~mm}$ curve at $0.2 \mathrm{~m}$ altitude). This points to a certain surface-activity of the employed microplastic particles: particles sticking to the reservoir surface would 
enrich the local concentration at the surface, thus resulting in a higher particle concentration within the splash droplets as well.

\section{Discussion: estimating the annual amount of microplastics transitioning from global oceans into the atmosphere due to impacting raindrops}

Based on our simulation results, we can provide an orderof-magnitude estimate for the global annual amount of microplastics transitioning from the oceans into the atmosphere due to raindrops. To guarantee reproducibility, our model assumes single, isolated raindrop impacts on a perfectly flat ocean surface. For this approximation, we use straight impacts only. We are aware that this represents an idealized scenario compared to what would be observed in field experiments. Given the robustness of our results even for oblique impacts as shown in Fig. 10, we are confident that this does not represent a major limitation.

We will proceed in three steps: in the first step, we obtain the number of raindrops as function of their size depending on the rain rate based on known experimental data. In the second step, using our simulations, we can predict the number of microplastic particles ejected into the air per square kilometer per hour, again depending on the rain fall rate and wind speed. In the final step, we provide an estimate of the global annual amount of microplastic transported into the atmosphere due to impacting raindrops.

\section{How many raindrops of which size are present in nature?}

As shown in section "How raindrop diameter affects microplastics transport", the amount of transported microplastic strongly depends on the diameter of the impacting raindrop. As a first step, it is therefore necessary to understand how the total amount of precipitation water is distributed across different raindrop sizes for different rain rates. We approximate the distribution by the Marshall-Palmer model [31, 33]: By number, small raindrops are exponentially more frequent than large raindrops, following

$$
N(d)=\Lambda e^{-\Lambda d}
$$

whereby $\Lambda=4.1\left(\frac{\mathcal{R}}{\frac{\mathrm{mm}}{\mathrm{h}}}\right)^{-0.21} \frac{1}{\mathrm{~mm}}$ is a parameter depending on the rainfall rate $\mathcal{R}$ in $\frac{\mathrm{mm}}{\mathrm{h}}$ and $d$ is the raindrop diameter in mm. Equation (13) is normalized. In order to reasonably estimate how the total precipitation volume splits up upon differently sized raindrops, we discretize the raindrop size distribution (13) to discrete raindrop diameter ranges. The probability to find a raindrop in the interval

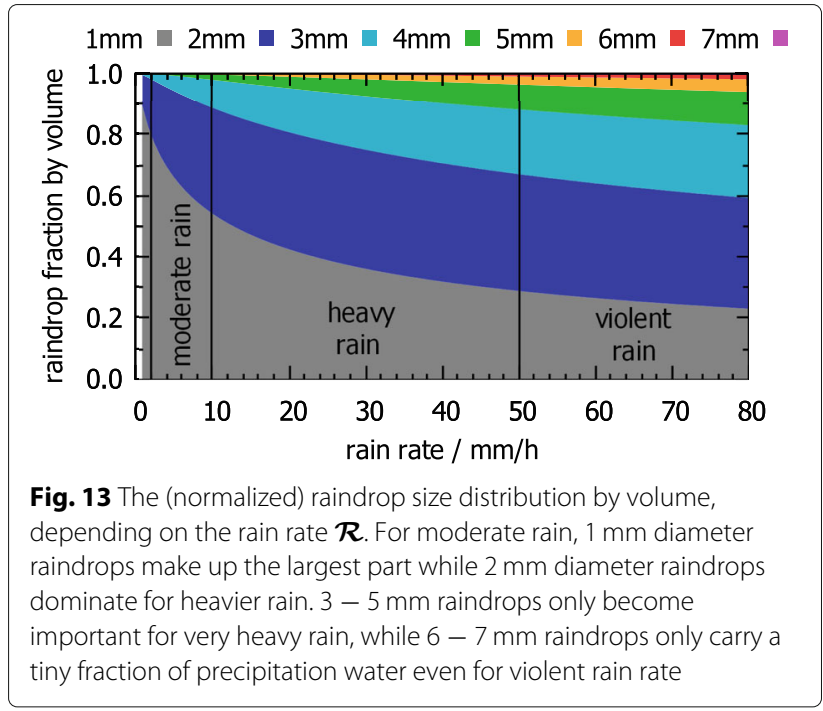

[ $d-0.5 \mathrm{~mm}, d+0.5 \mathrm{~mm}]$ then is:

$$
\begin{aligned}
p(d) & =\int_{d-0.5 \mathrm{~mm}}^{d+0.5 \mathrm{~mm}} N\left(d^{\prime}\right) \mathrm{d} d^{\prime} \\
& =\left[-e^{-\Lambda d^{\prime}}\right]_{d-0.5 \mathrm{~mm}}^{d+0.5 \mathrm{~mm}} \\
& =2 e^{-\Lambda d} \sinh \left(\frac{\Lambda}{2} \mathrm{~mm}\right)
\end{aligned}
$$

Using $p(d)$, we estimate how a given precipitation volume $V_{\text {pre }}$ splits up upon differently sized raindrops with $d \in\{1,2,3, \ldots, 7\} \mathrm{mm}$. The volume of a single raindrop is denoted as $V(d)=\frac{\pi}{6} d^{3}$. Computing the products $p(d)$. $V(d)$ and normalizing results yields the volume fractions associated with the raindrop diameters ${ }^{4}$ :

$$
p_{V}(d) \approx \frac{p(d) \cdot V(d)}{\sum_{d=1,2, \ldots, 7 \mathrm{~mm}} p(d) V(d)}
$$

$p_{V}(d)$ is plotted in Fig. 13 and used to calculate the precipitation volume for every raindrop diameter interval. We then divide by the volume $V(d)$ of a single raindrop and this way obtain the number of raindrops $N(d)$ for each raindrop diameter interval separately. The size distribution of raindrops is key for the estimate because raindrops of different size have different impact dynamics and generate vastly different amounts of spray droplets.

\section{Local estimate for the number of transitioning microplastic particles}

In the second step, we determine the airborne time of spray droplets based on atmospheric updraft by integrating their 3D trajectories with an additional vertical wind velocity offset $u_{\text {updraft }}$ as detailed in section "Trajectory of spray droplets: maximum altitude, airborne time and

\footnotetext{
${ }^{4}$ This assumes that the entire precipitation volume $V_{\text {pre }}$ is within the diameter range $\mathrm{d} \in[0.5 \mathrm{~mm}, 7.5 \mathrm{~mm}]$.
} 
pickup by wind" above. Figure 14 (a) shows that without updrafts, airborne time is less than $1 \mathrm{~s}$ and atmospheric uptake is considered negligible. Although the typical mean surface wind speed over the ocean is in the order of $8 \pm 4 \frac{\mathrm{m}}{\mathrm{s}}$ [103, figure 2], the vertical updraft velocity close to the ground is $\lesssim 1 \frac{\mathrm{m}}{\mathrm{s}}$ [57]. At this vertical wind velocity, all droplets smaller than $0.26 \mathrm{~mm}$ diameter have diverging airborne time (Fig. 14 (b)), i.e. they are taken up by the atmosphere. Alternatively, if the airborne time remains finite, but droplets evaporate before falling back to the surface, we also consider the contained microplastic particles

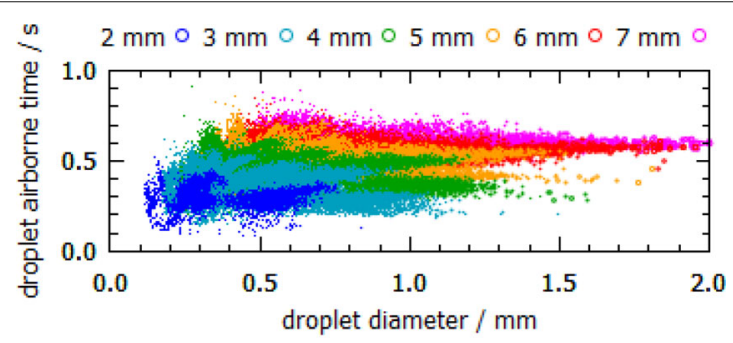

(a) Droplet airborne time without updraft.

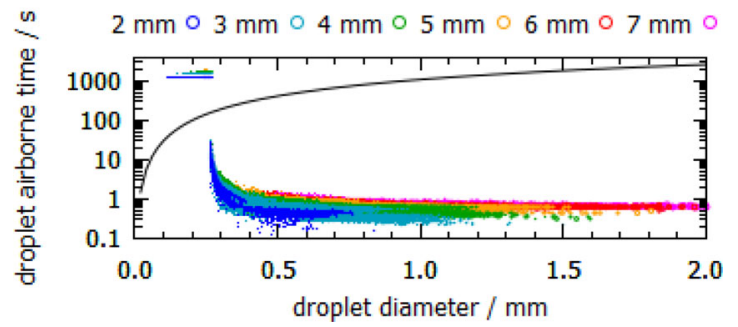

(b) Droplet airborne time with $1 \frac{\mathrm{m}}{\mathrm{s}}$ vertical updraft.

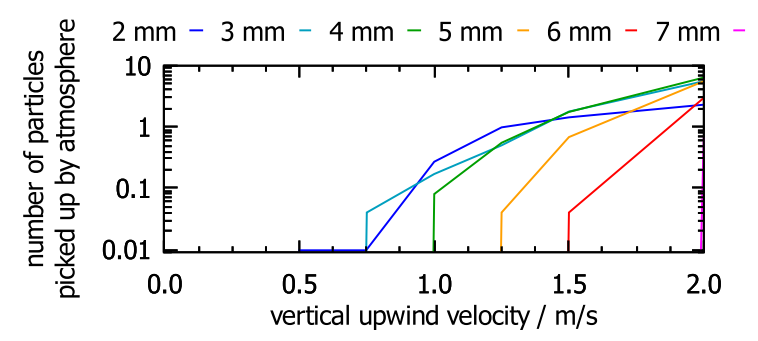

(c) Number of particles with diverging airborne time which are consequently picked up by wind and transfered to the atmosphere.

Fig. 14 (a) Airborne time of spray droplets without updraft is in the order of half a second. No droplets are picked up by the atmosphere. (b) With $1 \frac{\mathrm{m}}{\mathrm{s}}$ vertical updraft velocity, all droplets smaller than $0.26 \mathrm{~mm}$ diameter have diverging airborne time (capped at finite values so that the data points are visible in the diagram). The black curve represents the lifetime (time until full evaporation, Eq. (11)) of droplets depending on diameter. If the airborne time is larger than the lifetime, the droplets are considered picked up by the atmosphere. (c) The number of picked up microplastic particles per raindrop impact increases with vertical updraft velocity. Numbers are given for an initial concentration of 5000 microplastic particles per $\mathrm{cm}^{3}$ in the sea water as used in our simulations. Additional figures and data in Figure S24 and Table S3 as being taken up by the atmosphere. Together, these two criteria provide a very clear threshold for which droplets are picked up or fall back down, because all droplets above the lifetime curve have diverging airborne time as a function of updraft velocity (Fig. 14 (c)). Again, we emphasize that assuming constant updraft velocity is a simplified model and in nature the turbulent air movement is much more complicated.

The number of ejected particles per raindrop impact is rescaled with the concentration of microplastics at the surface, from the 5000 particles per $\mathrm{cm}^{3}$ in our simulations to a realistic value for the global average microplastic concentration in ocean surface waters, for which we choose 0.0029 particles per $\mathrm{cm}^{3}$ (2.9 particles per liter) as measured at a depth of $5 \mathrm{~m}$ [54]. This value is in line with other measurements across the Atlantic Ocean which gave concentrations between $0.99-7.00$ particles per liter at a depth of $10 \mathrm{~m}$ [3]. Coincidentally, the size of the microplastics detected by [54] is in the range of $10-600 \mu \mathrm{m}$ and the size detected by [3] is between $32-651 \mu \mathrm{m}$ with a mean of $81 \mu \mathrm{m}$, very close to the particle sizes used in our simulations. However these measurements likely undercount particles smaller than $10 \mu \mathrm{m}$ as these are increasingly difficult to detect.

Direct trawl measurements of the microplastic concenteration at the sea surface [20-22] are not suitable here for two reasons: Firstly, these are measurements per area and to convert to a volumetric concentration, one would have to make an assumption about the mean submersion depth. Secondly and more importantly, the trawls have a mesh size of $\approx 330 \mu \mathrm{m}$, which is insufficient to detect the much more numerous tiny particles. However these trawl measurements give a good indication about the large local and temporal variation in concentration, spanning across more than two orders of magnitude [3, 20-22].

Next, we multiply the number of raindrops $N(d)$ by the number of ejected particles per raindrop impact $N_{p}(d)$ to obtain the number of ejected particles for every raindrop diameter. Summing over all diameters between 1 and $7 \mathrm{~mm}$, we obtain the amount of transitioning particles per square kilometer per hour as function of the rain rate which is shown in Fig. 15. We find that both local rain rate and wind speed strongly affect particle uptake (Fig. 15). It is interesting that $2-3 \mathrm{~mm}$ diameter raindrops contribute the vast majority to the transitioning particles with larger raindrops only having very minor contribution at violent rain rate (Fig. 15 (a)). During heavy storms, anything detached from the sea surface may be carried across vast distances by wind, including droplets, particles $[17$, $18]$ and even various sea creatures [104, 105]. During mild weather conditions on the other hand, most spray droplets may fall back to the surface before significant atmospheric transport has happened. 


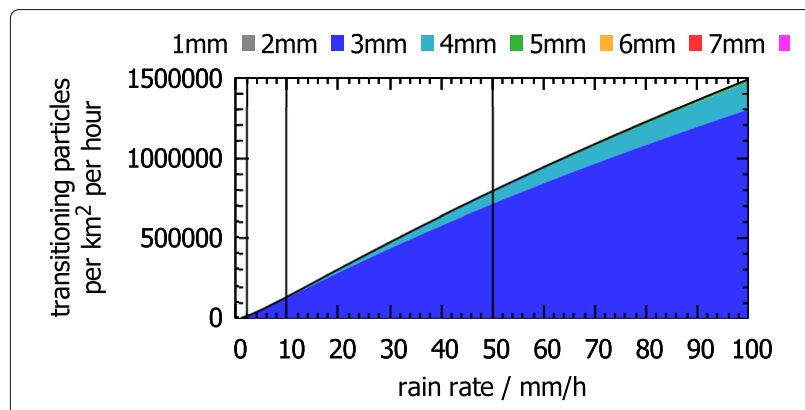

(a) Contribution of different raindrop diameters to particle uptake for $1 \frac{\mathrm{m}}{\mathrm{s}}$ vertical updraft velocity.

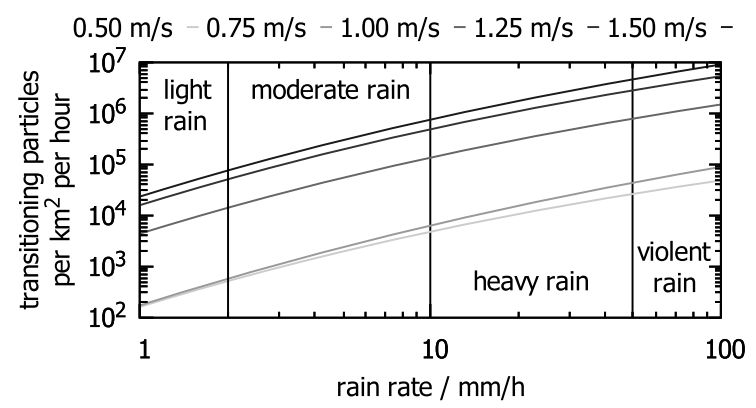

(b) Number of microplastic particles depending on rain rate for various vertical updraft velocities.

Fig. 15 (a) The number of microplastic particles transitioning from the oceans into the atmosphere per $\mathrm{km}^{2}$ per hour depending on rain rate for $1 \frac{\mathrm{m}}{\mathrm{s}}$ vertical updraft velocity. The contribution of different raindrop sizes to particle transport is illustrated by the colored areas. With rising rain rate, the number of transitioning particles increases as both the number of raindrops and the fraction of larger raindrops become larger. (b) Different vertical updraft velocity strongly affects the number of transitioning particles. For less than $0.5 \frac{\mathrm{m}}{\mathrm{s}}$, no particles get picked up by the atmosphere

\section{Global estimate for the number of transitioning microplastic particles}

In the final step, we use the above considerations to provide a rough estimate for the global annual amount of microplastic transferred to the atmosphere by impacting raindrops.

We first observe that the rain rate itself follows an exponential distribution, where low rain rate is much more frequent and high rain rate is much less frequent. We use the (normalized) Rice-Holmberg model [56]

$$
\begin{aligned}
P_{\mathrm{RH}}(\mathcal{R})= & \frac{1}{1.80273}\left(0.03 e^{-0.03 \mathcal{R}}\right. \\
& \left.+\frac{1-\beta}{5}\left(e^{-0.258 \mathcal{R}}+1.86 e^{-1.63 \mathcal{R}}\right)\right)
\end{aligned}
$$

with $\beta=0.2$. According to [55], the average precipitation on the ocean is $2.89 \pm 0.29 \frac{\mathrm{mm}}{\mathrm{day}}$. Global oceans cover a surface of $3.619 \cdot 10^{14} \mathrm{~m}^{2}$, so the total annual precipitation volume on the oceans is $V_{\text {pre,total }}=3.82 \cdot 10^{14} \mathrm{~m}^{3}$. For estimating the annual global amount, we set this value as $V_{\text {pre }}$ in our calculations detailed above (Figure S25). We then weight the curves in Figure S25 with the Rice-Holmberg rain rate distribution (Eq. (18), numeric integration with trapezoidal rule). Finally, we assume that vertical wind direction is upward and downward half of the time and no particles transition for downward wind velocity, giving us another factor $1 / 2$. We then find that, depending on which vertical updraft velocity we assume, $7.0 \cdot 10^{13}\left(0.50 \frac{\mathrm{m}}{\mathrm{s}}\right)$, $1.0 \cdot 10^{14}\left(0.75 \frac{\mathrm{m}}{\mathrm{s}}\right), 2.0 \cdot 10^{15}\left(1.00 \frac{\mathrm{m}}{\mathrm{s}}\right), 7.3 \cdot 10^{15}\left(1.25 \frac{\mathrm{m}}{\mathrm{s}}\right)$ or $1.2 \cdot 10^{16}\left(1.50 \frac{\mathrm{m}}{\mathrm{s}}\right)$ microplastic particles may transition from global oceans into the atmosphere every year. Because $0.50-0.75 \frac{\mathrm{m}}{\mathrm{s}}$ is already rather large for vertical updraft velocity close to the ground [57], our estimation gives us a clear value for the upper bound, a hundred trillion $\left(10^{14}\right)$ microplastic particles per year. For vertical updraft velocities below $0.50 \frac{\mathrm{m}}{\mathrm{s}}$, based on our definition of atmospheric suspension, no particles transition, so we cannot confidently provide a lower bound for the estimate.

The uncertainties of this estimate are located in the local sea surface microplastic concentration, but also in other simplifications of our model such as assuming mean updraft velocity instead of turbulence. In future research, we envision to use more precise spatially resolved wind and microplastic distribution data using for example satellite measurements [106], such as currently investigated in the TOPIOS project $[5,107,108]$, to further narrow down this estimate. This refinement should also include the effect that microplastic particles right after transition to the atmosphere may collide with subsequent raindrops and therefore may be washed out of the atmosphere again leading to a reduction of total transport.

\section{Conclusions}

We investigated and quantified microplastic particle transport across the water-air interface during raindrop impacts on sea water in great detail using numerical simulations and laboratory experiments.

Depending on raindrop size, each impact ejects in the order of 100 splash droplets into the air with typical vertical velocities of up to $10 \frac{\mathrm{m}}{\mathrm{s}}$, allowing them to reach altitudes of up to about $80 \mathrm{~cm}$ above the sea surface.

Our key result is that the particle concentration in these ejected droplets is about $85 \%$ of that in the sea water. This means that there is no 'filter effect' holding particles back and that the fluid from the raindrop, even if it is devoid of particles, is mainly engulfed into the sea while the ejected fluid mainly consists of sea water. We further found that the particle density has negligible influence on the impact dynamics, since gravity-related effects only play a minor role during the short time scale of the impact. The origin of the ejected particles has been identified as a circular region around the impact site very close to the sea surface. No particles are ejected from regions deeper than approximately half the radius of the raindrop. 
For raindrops impacting the surface at an angle, e.g. due to wind, part of the raindrop fluid is redirected into the spray and droplets can reach slightly higher altitudes. The overall mechanism of microplastic transport is nevertheless operative also for oblique drop impacts.

Our laboratory experiments of artificially produced raindrops with a well-defined size and velocity on a reservoir filled with high concentrations of microplastic particles are in good agreement with the simulation predictions. The experiments clearly confirm that microplastic particles are contained in the ejected spray droplets.

Based on our simulations of a single raindrop event, assuming an average microplastic particle concentration of 2.9 particles per liter at the ocean surface, we estimate that a realistic upper bound for the annual number of microplastic particles transitioning during rainfall from global oceans into the atmosphere is a hundred trillion $\left(10^{14}\right)$. This estimate contains a number of uncertainties such as microplastic concentration at the ocean surface, calling for additional research to narrow this range further down.

\section{Supplementary Information}

The online version contains supplementary material available at https://doi.org/10.1186/s43591-021-00018-8.

Additional file 1: SI - Ejection of marine microplastics by raindrops: a computational and experimental study.

Additional file 2: figure-11.mp4

Additional file 3: figure-12a.mp4

Additional file 4: figure-S19f.mp4

\section{Acknowledgements}

We acknowledge support through the computational resources provided by the Bavarian Polymer Institute. We acknowledge the NVIDIA Corporation for donating a Titan Xp GPU for our research. ML acknowledges support from the ENB Biological Physics.

\section{Authors' contributions}

$\mathrm{ML}$ and FH contributed the software used for the simulations. ML and SG contributed design of the study. ML contributed to data analysis and evaluation. ML, SG, LMO and AH contributed writing and literature research. $\mathrm{ML}$ conducted the simulations. $\mathrm{LMO}$ and $\mathrm{AH}$ conducted the experiments. The authors read and approved the final manuscript.

\section{Funding}

This study was funded by the Deutsche Forschungsgemeinschaft (DFG, German Research Foundation) - Project Number 391977956 - SFB 1357. Open Access funding enabled and organized by Projekt DEAL.

\section{Availability of data and materials}

The data sets used and/or analyzed during the current study are publicly available on Zenodo and the simulation source code is available from the corresponding author upon reasonable request.

\section{Declarations}

\section{Competing interests}

The authors declare that they have no competing interests.

\section{Author details}

${ }^{1}$ Biofluid Simulation and Modeling - Theorethische Physik VI, University of Bayreuth, Bayreuth, Germany. ${ }^{2}$ Technischer Umweltschutz, Fachgebiet
Umweltchemie und Luftreinhaltung, Technische Universität Berlin, Berlin, Germany.

Received: 18 March 2021 Accepted: 21 September 2021

Published online: 12 November 2021

References

1. Rochman CM. Microplastics research-from sink to source. Science. 2018;360(6384):28-9.

2. Cózar A, Echevarría F, González-Gordillo Jl, Irigoien X, Úbeda B, Hernández-León S, Palma ÁT, Navarro S, García-de-Lomas J, Ruiz A, et al. Plastic debris in the open ocean. Proc Natl Acad Sci. 2014;111(28): 10239-44.

3. Pabortsava K, Lampitt RS. High concentrations of plastic hidden beneath the surface of the atlantic ocean. Nat Commun. 2020;11(1):1-11.

4. Woodall LC, Sanchez-Vidal A, Canals M, Paterson GL, Coppock R, Sleight V, Calafat A, Rogers AD, Narayanaswamy BE, Thompson RC. The deep sea is a major sink for microplastic debris. Roy Soc Open Sci. 2014;1 (4):140317.

5. Onink V, Jongedijk CE, Hoffman MJ, van Sebille E, Laufkötter C. Global simulations of marine plastic transport show plastic trapping in coastal zones. Environ Res Lett. 2021;16(6):064053.

6. Allen $S$, Allen D, Phoenix VR, Le Roux G, Jiménez PD, Simonneau A, Binet S, Galop D. Atmospheric transport and deposition of microplastics in a remote mountain catchment. Nat Geosci. 2019;12(5):339-44.

7. Trainic M, Flores JM, Pinkas I, Pedrotti ML, Lombard F, Bourdin G, Gorsky G, Boss E, Rudich Y, Vardi A, et al. Airborne microplastic particles detected in the remote marine atmosphere. Commun Earth Environ. 2020;1 (1):1-9.

8. Tirelli V, Suaria G, Lusher AL. In: Rocha-Santos T, Costa M, Mouneyrac C, editors. Microplastics in Polar Samples. Cham: Springer; 2020, pp. 1-42. https://doi.org/10.1007/978-3-030-10618-8_.

9. Napper IE, Davies BF, Clifford H, Elvin S, Koldewey HJ, Mayewski PA, Miner KR, Potocki M, Elmore AC, Gajurel AP, et al. Reaching new heights in plastic pollution-preliminary findings of microplastics on mount everest. One Earth. 2020;3(5):621-30.

10. Lhuissier $\mathrm{H}$, Villermaux E. Bursting bubble aerosols. J Fluid Mech. 2012;696:5.

11. Ghabache E, Séon T. Size of the top jet drop produced by bubble bursting. Phys Rev Fluids. 2016;1 (5):051901.

12. Berny $A$, Deike $L$, Séon $T$, Popinet $S$. Role of all jet drops in mass transfer from bursting bubbles. Phys Rev Fluids. 2020;5(3):033605.

13. Masry M, Rossignol $\mathrm{S}$, Roussel $\mathrm{BT}$, Bourgogne $\mathrm{D}$, Bussière $\mathrm{P}-\mathrm{O}, \mathrm{R}^{\prime}$ mili $B$ Wong-Wah-Chung P. Experimental evidence of plastic particles transfer at the water-air interface through bubble bursting. Environ Pollut. 2021;280:116949.

14. Veron F. Ocean spray. Ann Rev Fluid Mech. 2015;47:507-38.

15. Blanchard DC. Sea-to-air transport of surface active material. Science. 1964;146(3642):396-7.

16. Blanchard DC. Jet drop enrichment of bacteria, virus, and dissolved organic material. Pure Appl Geophys. 1978;1 16(2-3):302-8.

17. Quinn JA, Steinbrook RA, Anderson JL. Breaking bubbles and the water-to-air transport of particulate matter. Chem Eng Sci. 1975;30(9): 1177-84.

18. de Leeuw G, Andreas EL, Anguelova MD, Fairall CW, Lewis ER, O'Dowd C, Schulz M, Schwartz SE. Production flux of sea spray aerosol. Rev Geophys. 2011;49(2):13-39.

19. O'Dowd CD, Smith MH, Consterdine IE, Lowe JA. Marine aerosol, sea-salt, and the marine sulphur cycle: A short review. Atmos Environ. 1997;31(1):73-80

20. Lacerda ALdF, Rodrigues LdS, Van Sebille E, Rodrigues FL, Ribeiro L, Secchi ER, Kessler F, Proietti MC. Plastics in sea surface waters around the antarctic peninsula. Sci Rep. 2019;9(1):1-12.

21. Naidoo T, Glassom D. Sea-surface microplastic concentrations along the coastal shelf of KwaZulu-Natal, South Africa. Mar Pollut Bull. 2019;149: 110514

22. Gajšt T, Bizjak T, Palatinus A, Liubartseva S, Kržan A. Sea surface microplastics in Slovenian part of the Northern Adriatic. Mar Pollut Bull. 2016;113(1-2):392-9. 
23. Suaria G, Achtypi A, Perold V, Lee JR, Pierucci A, Bornman TG, Aliani S, Ryan PG. Microfibers in oceanic surface waters: A global characterization. Sci Adv. 2020;6(23):8493.

24. Robinson T-B, Giebel H-A, Wurl O. Riding the plumes: characterizing bubble scavenging conditions for the enrichment of the sea-surface microlayer by transparent exopolymer particles. Atmosphere. 2019;10(8):454.

25. Anderson ZT, Cundy AB, Croudace IW, Warwick PE, Celis-Hernandez O, Stead JL. A rapid method for assessing the accumulation of microplastics in the sea surface microlayer (SML) of estuarine systems. Sci Rep. 2018;8(1):1-11.

26. Stead JL, Cundy AB, Hudson MD, Thompson CE, Williams ID, Russell $A E$, Pabortsava K. Identification of tidal trapping of microplastics in a temperate salt marsh system using sea surface microlayer sampling. Sci Rep. 2020;10(1):1-10.

27. Song YK, Hong SH, Jang M, Kang J-H, Kwon OY, Han GM, Shim WJ. Large accumulation of micro-sized synthetic polymer particles in the sea surface microlayer. Environ Sci Technol. 2014;48(16):9014-21.

28. Allen S, Allen D, Moss K, Le Roux G, Phoenix VR, Sonke JE. Examination of the ocean as a source for atmospheric microplastics. PloS ONE. 2020;15(5):0232746.

29. Porcù F, D'adderio LP, Prodi F, Caracciolo C. Effects of altitude on maximum raindrop size and fall velocity as limited by collisional breakup. J Atmos Sci. 2013;70(4):1129-34.

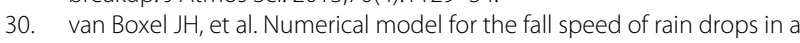
rain fall simulator. In: Workshop on Wind and Water Erosion; 1997. p. 77-85.

31. Marshall JS, Palmer WMK. The distribution of raindrops with size. J Meteorol. 1948;5(4):165-6.

32. Spilhaus AF. Raindrop size, shape and falling speed. J Meteorol. 1948;5(3):108-10.

33. Villermaux E, Bossa B. Single-drop fragmentation determines size distribution of raindrops. Nat Phys. 2009;5(9):697-702.

34. Rein M. Phenomena of liquid drop impact on solid and liquid surfaces. Fluid Dyn Res. 1993;12(2):61.

35. Rein M. The transitional regime between coalescing and splashing drops. J Fluid Mech. 1996;306:145-65.

36. Fedorchenko Al, Wang A-B. On some common features of drop impact on liquid surfaces. Phys Fluids. 2004;16(5):1349-65.

37. Bisighini A, Cossali GE. High-speed visualization of interface phenomena: single and double drop impacts onto a deep liquid layer. J Vis. 2011;14(2):103-10.

38. Myagkov N, Shumikhin T. Modeling of high-velocity impact ejecta by experiments with a water drop impacting on a water surface. Acta Mech. 2016;227(10):2911-24.

39. Leng $\sqcup$. Splash formation by spherical drops. J Fluid Mech. 2001;427: 73-105.

40. Ray B, Biswas G, Sharma A. Regimes during liquid drop impact on a liquid pool. J Fluid Mech. 2015;768:492-523.

41. Michon G-J, Josserand C, Séon T. Jet dynamics post drop impact on a deep pool. Phys Rev Fluids. 2017;2(2):023601.

42. Ding Q, Wang T, Che Z. Two jets during the impact of viscous droplets onto a less-viscous liquid pool. Phys Rev E. 2019;100(5):053108.

43. Gielen MV, Sleutel P, Benschop J, Riepen M, Voronina V, Visser CW, Lohse D, Snoeijer JH, Versluis M, Gelderblom H. Oblique drop impact onto a deep liquid pool. Phys Rev Fluids. 2017;2(8):083602.

44. Oguz HN, Prosperetti A. Bubble entrainment by the impact of drops on liquid surfaces. J Fluid Mech. 1990;219:143-79.

45. Motzkus C, Géhin E, Gensdarmes F. Study of airborne particles produced by normal impact of millimetric droplets onto a liquid film. Exp Fluids. 2008;45(5):797.

46. Murphy DW, Li C, d'Albignac V, Morra D, Katz J. Splash behaviour and oily marine aerosol production by raindrops impacting oil slicks. J Fluid Mech. 2015;780:536.

47. Medwin H, Nystuen JA, Jacobus PW, Ostwald LH, Snyder DE. The anatomy of underwater rain noise. J Acoust Soc Am. 1992;92(3):1613-23.

48. Prosperetti A, Oguz HN. The impact of drops on liquid surfaces and the underwater noise of rain. Ann Rev Fluid Mech. 1993;25(1):577-602.

49. Wang $P$, Pruppacher $H$. Acceleration to terminal velocity of cloud and raindrops. J Appl Meteorol. 1977;16(3):275-80.
50. Guo Y, Lian Y. High-speed oblique drop impact on thin liquid films. Phys Fluids. 2017;29(8):082108.

51. Lehmann M. High Performance Free Surface LBM on GPUs. Master's thesis: Universität Bayreuth, Fakultät für Mathematik, Physik und Informatik; 2019. https://epub.uni-bayreuth.de/5400/.

52. Lehmann M, Gekle S. Analytic solution to the piecewise linear interface construction problem and its application in curvature calculation for volume-of-fluid simulation codes. arXiv preprint arXiv:2006.12838. 2020;2006:12838.

53. Häusl F. MPI-based multi-GPU extension of the Lattice Boltzmann Method. Bachelor's thesis: Universität Bayreuth, Fakultät für Mathematik, Physik und Informatik; 2019. https://epub.uni-bayreuth.de/5689/.

54. Choy CA, Robison BH, Gagne TO, Erwin B, Firl E, Halden RU, Hamilton JA, Katija K, Lisin SE, Rolsky C, et al. The vertical distribution and biological transport of marine microplastics across the epipelagic and mesopelagic water column. Sci Rep. 2019;9(1):1-9.

55. Adler RF, Gu G, Sapiano M, Wang J-J, Huffman GJ. Global precipitation: Means, variations and trends during the satellite era (1979-2014). Surv Geophys. 2017;38(4):679-99.

56. Rice $P$, Holmberg N. Cumulative time statistics of surface-point rainfall rates. IEEE Trans Commun. 1973;21(10):1131-6.

57. Ouwersloot HG, de Roode SR, Bosveld FC, Kroon PS. Vertical wind velocity observations at the Cabauw tower. In: 19th Symposium on Boundary Layers and Turbulence, American Meteorological Society, 2-6 August 2010, Keystone, Colorado, USA; 2010. p. 1-2.

58. Wittmann M. Hardware-effiziente, hochparallele Implementierungen von Lattice-Boltzmann-Verfahren für komplexe Geometrien. PhD thesis: Friedrich-Alexander-Universität Erlangen-Nürnberg (FAU); 2016. https:// nbn-resolving.org/urn:nbn:de:bvb:29-opus4-74586.

59. Wittmann M. Hardware-effiziente, hochparallele implementierungen von lattice-boltzmann-verfahren für komplexe geometrien. 2016.

60. Delbosc N, Summers JL, Khan A, Kapur N, Noakes CJ. Optimized implementation of the lattice Boltzmann method on a graphics processing unit towards real-time fluid simulation. Comput Math Appl. 2014;67(2):462-75

61. Herschlag G, Lee S, Vetter JS, Randles A. GPU data access on complex geometries for D3Q19 lattice Boltzmann method. In: 2018 IEEE International Parallel and Distributed Processing Symposium (IPDPS). Vancouver: IEEE International Parallel and Distributed Processing Symposium (IPDPS); 2018. p. 825-34.

62. Mawson MJ, Revell AJ. Memory transfer optimization for a lattice Boltzmann solver on Kepler architecture nVidia GPUs. Comput Phys Commun. 2014;185(10):2566-74.

63. Wittmann M, Zeiser T, Hager G, Wellein G. Comparison of different propagation steps for lattice Boltzmann methods. Comput Math Appl. 2013;65(6):924-35

64. Kuznik F, Obrecht C, Rusaouen G, Roux J-J. LBM based flow simulation using GPU computing processor. Comput Math Appl. 2010;59(7): 2380-92.

65. Obrecht C, Kuznik F, Tourancheau B, Roux J-J. Multi-GPU implementation of the lattice Boltzmann method. Comput Math Appl. 2013;65(2):252-61

66. Krüger T, Kusumaatmaja H, Kuzmin A, Shardt O, Silva G, Viggen EM. The lattice Boltzmann method. Springer Int Publ. 2017;10(978-3):4-15.

67. Chapman S, Cowling TG, Burnett D. The Mathematical Theory of Non-uniform Gases: an Account of the Kinetic Theory of Viscosity, Thermal Conduction and Diffusion in Gases. Cambridge, UK: Cambridge university press; 1990.

68. Purqon A, et al. Accuracy and numerical stabilty analysis of lattice Boltzmann method with multiple relaxation time for incompressible flows. In: Journal of Physics: Conference Series. Bristol: IOP Publishing; 2017. p. 012035.

69. Cui X, Wang Z, Yao X, Liu M. A coupled two-relaxation-time lattice boltzmann-volume penalization method for flows past obstacles. arXiv preprint arXiv:1901.08766. 2019;1901:08766

70. Guo Z, Shu C, Vol. 3. Lattice Boltzmann Method and Its Applications in Engineering. Singapore: World Scientific; 2013.

71. Saito S, Abe Y, Koyama K. Lattice boltzmann modeling and simulation of liquid jet breakup. Phys Rev E. 2017;96(1):013317.

72. Kuzmin A, Guo Z, Mohamad A. Simultaneous incorporation of mass and force terms in the multi-relaxation-time framework for lattice 
Boltzmann schemes. Philos Trans R Soc A Math Phys Eng Sci. 2011;369(1944):2219-27

73. Guo Z, Zheng C, Shi B. Discrete lattice effects on the forcing term in the lattice Boltzmann method. Phys Rev E. 2002;65(4):046308.

74. Pohl T. High Performance Simulation of Free Surface Flows Using the Lattice Boltzmann Method. Erlangen, Germany: Verlag Dr. Hut; 2008.

75. Donath S. Wetting Models for a Parallel High-performance Free Surface Lattice Boltzmann Method: Benetzungsmodelle Für Eine Parallele Lattice-Boltzmann-Methode Mit Freien Oberflächen. Erlangen, Germany: Verlag Dr. Hut; 2011.

76. Körner C, Thies M, Hofmann T, Thürey N, Rüde U. Lattice boltzmann model for free surface flow for modeling foaming. J Stat Phys. 2005;121(1-2):179-96.

77. Thürey N, Körner C, Rüde U. Interactive free surface fluids with the lattice Boltzmann method. PhD thesis: Technical Report05-4. University of Erlangen-Nuremberg, Germany. Citeseer; 2005. http://www.thuerey. de/ntoken/download/nthuerey_050607_tr1rtlbm.pdf.

78. Schreiber M, Neumann DTMP. GPU based simulation and visualization of fluids with free surfaces. PhD thesis: Technische Universität München 2010. https://www.martin-schreiber.info/data/research_diplomathesis/ thesis_2010_06_08.pdf.

79. Janßen C, Krafczyk M. Free surface flow simulations on GPGPUs using the LBM. Comput Math Appl. 2011;61(12):3549-63.

80. Bogner S, Rüde U, Harting J. Curvature estimation from a volume-of-fluid indicator function for the simulation of surface tension and wetting with a free-surface lattice boltzmann method. Phys Rev E. 2016;93(4):043302.

81. Youngs DL. An interface tracking method for a 3D Eulerian hydrodynamics code. Atom Weapons Res Establishment (AWRE) Tech Rep. 1984;44(92):35.

82. Scardovelli R, Zaleski S. Analytical relations connecting linear interfaces and volume fractions in rectangular grids. J Comput Phys. 2000;164(1): 228-37.

83. Kawano A. A simple volume-of-fluid reconstruction method for three-dimensional two-phase flows. Comput Fluids. 2016;134:130-45.

84. Bourke P. Polygonising a scalar field. 1994. http://paulbourke.net/ geometry/polygonise/.

85. Peskin CS. The immersed boundary method. ANU. 2003;11:479-517.

86. Krüger T. Introduction to the immersed boundary method. In: LBM Workshop, Edmonton; 2011.

87. Bourke P. Interpolation methods. Misc Projection Model Rendering. 1999;1(10):.

88. Hamuraru A. Atomic operations for floats in OpenCL - improved. 2016. https://streamhpc.com/blog/2016-02-09/atomic-operations-for-floatsin-opencl-improved/. Accessed 15 Nov 2019.

89. Frijters $S$, Krüger T, Harting J. Parallelised Hoshen?Kopelman algorithm for lattice-Boltzmann simulations. Comput Phys Commun. 2015;189: 92-8.

90. Holterman H, Vol. 2012. Kinetics and Evaporation of Water Drops in Air. Wageningen, Netherlands: Citeseer; 2003.

91. Morrison FA. An Introduction to Fluid Mechanics. New York, USA: Cambridge University Press; 2013.

92. Feng Z-G, Michaelides EE. Drag coefficients of viscous spheres at intermediate and high Reynolds numbers. J Fluids Eng. 2001;123(4): 841-9.

93. Beccario C. earth.nullschool.net. 2021. https://earth.nullschool.net/. Accessed 25 June 2021.

94. The Engineering ToolBox. Air - Density, Specific Weight and Thermal Expansion Coefficient at Varying Temperature and Constant Pressures. 2020. https://www.engineeringtoolbox.com/air-absolute-kinematicviscosity-d_601.html. Accessed 23 Aug 2020.

95. The Engineering ToolBox. Air - Dynamic and Kinematic Viscosity. 2020. https://www.engineeringtoolbox.com/air-absolute-kinematic-viscosityd_601.html. Accessed 29 July 2020.

96. ITTC Specialist Committee, et al. ITTC-Recommended Procedures Fresh Water and Seawater Properties. In: Proceedings of ITTC 2011. ITTC; 2011. https://ittc.info/media/4048/75-02-01-03.pdf.

97. The International Association for the Properties of Water and Steam. Guideline on the Surface Tension of Seawater, vol. G14-19: IAPWS; 2019. http://www.iapws.org/relguide/Seawater-Surf.pdf.
98. Bergmann R, van der Meer D, Gekle S, van der Bos A, Lohse D. Controlled impact of a disk on a water surface: cavity dynamics. J Fluid Mech. 2009;633:381.

99. Eshraghi J, Jung S, Vlachos PP. To seal or not to seal: The closure dynamics of a splash curtain. Phys Rev Fluids. 2020;5(10):104001.

100. Gekle S, Gordillo JM, van der Meer D, Lohse D. High-speed jet formation after solid object impact,. Phys Rev Lett. 2009;102(3):034502.

101. Einstein A. Eine neue bestimmung der moleküldimensionen: ETH Zurich 1905.

102. Haines BM, Mazzucato AL. A proof of einstein's effective viscosity for a dilute suspension of spheres. SIAM J Math Anal. 2012;44(3):2120-45.

103. Monahan $\mathrm{AH}$. The probability distribution of sea surface wind speeds. Part I: Theory and SeaWinds observations. J Climate. 2006;19(4):497-520.

104. MCATEE W. Showers of organic matter. Mon Weather Rev. 1917;45(5): 217-24.

105. Whitley GP. Rains of fishes in Australia. Aust Nat Hist. 1972;17:154-9.

106. Evans MC, Ruf CS. Toward the detection and imaging of ocean microplastics with a spaceborne radar. IEEE Trans Geosci Remote Sens. 20211-9. https://ieeexplore.ieee.org/document/9449485.

107. Wichmann D, Delandmeter $P$, van Sebille E. Influence of near-surface currents on the global dispersal of marine microplastic. J Geophys Res Oceans. 2019;124(8):6086-96.

108. Wichmann D, Delandmeter P, Dijkstra HA, van Sebille E. Mixing of passive tracers at the ocean surface and its implications for plastic transport modelling. Environ Res Commun. 2019;1(11):115001.

\section{Publisher's Note}

Springer Nature remains neutral with regard to jurisdictional claims in published maps and institutional affiliations.

\section{Submit your manuscript to a SpringerOpen ${ }^{\circ}$ journal and benefit from:}

- Convenient online submission

- Rigorous peer review

- Open access: articles freely available online

- High visibility within the field

- Retaining the copyright to your article

Submit your next manuscript at $>$ springeropen.com 